\title{
MULTIPLIER RINGS AND PRIMITIVE IDEALS
}

\author{
BY
}

JOHN DAUNS

The multiplier ring $M(A)$ of any ring without an identity is the biggest essential extension $A \rightarrow M(A)$ where the image of $A$ is an ideal (notation: $A \triangleleft M(A)$ ). Recently, $M(A)$ has received considerable attention. B. E. Johnson studied the multiplier for semigroups, rings, and topological algebras [7]. R. C. Busby used it for classifying extensions of $C^{*}$-algebras in [1], and in [2] to study the spectrum of an algebra. In [6], $M(A)$ is used from a different point of view; there the question when a $C^{*}$-algebra $A$ is an ideal in its second dual is considered.

$\S 1$ develops several useful properties of the multiplier for associative rings, including a characterization of the multiplier as the adjoint of a certain forgetful functor. Perhaps some of these considerations can be carried over to other categories, such as abelian or topological groups. In $\S 2$ an extension $A \triangleleft \hat{A}$ is considered. Not only the connection between Prim $A$-the primitive ideals of $A$-and Prim $\hat{A}$ is established, but also simultaneously and within the same framework, the correspondence between the associated regular maximal left ideals as well as the simple $A$ and $\hat{A}$-modules is completely described. No assumptions other than $A \triangleleft \hat{A}$ are imposed; an identity for $\hat{A}$ is not assumed. For this reason the above development might be of interest because some of the above mentioned results about $A \subset \hat{A}$ had been proved previously with $\hat{A}=M(A)$ for special kinds of rings, such as $C^{*}$-algebras, by using very special and frequently irrelevant properties of these rings. $\S 3$ deals with more special extensions of the form $\tilde{A}=S+A$, with $A \triangleleft \tilde{A}$, and $S$ a subring, where $S \cap A=\{0\}$ is not always assumed.

The first part of the paper has been written, as far as possible, so as to be selfcontained. However, in the remainder $A$ is specialized to a $C^{*}$-algebra and some familiarity with [4] (or [1] and [5]) is required. The center $R$ of $M(A)$ is called the centroid of $A$. In [4], Prim $(R+A)$ was described. In $\S 4$ a description of Prim $M(A)$ is given. For $M(A)$, just as was the case for $R+A$ in [4], another space of ideals, obtained as the complete regularization of the primitive ones, plays an even more important role. It also is described. It is shown that the primitive ideal space of the center of $A$ can be identified with a certain subset of ideals of the complete regularization of Prim $A$, thus generalizing a result of Busby [2]. The objective of the last section is to identify and characterize closed ideals $A_{2}$

Received by the editors July 26, 1968 and, in revised form, March 13, 1969. 
in $A \subset A_{2} \subset R+A$. With each of the three algebras is associated the regularization of its primitive ideals $M, M_{2}, M_{3}$ so that the three algebras are subdirect products

$$
A \subset \prod_{m \in M} \frac{A}{m}, \quad A_{2} \subset \prod_{m_{2} \in M_{2}} \frac{A_{2}}{m_{2}}, \quad A_{3} \subset \prod_{m_{3} \in M_{3}} \frac{A_{3}}{m_{3}} .
$$

Already in $\S 4$ a complete description of Prim $A_{2}$ is given, while in $\S 5$ the picture is completed by describing each $m_{2} \in M_{2}$ and each quotient $A_{2} / m_{2}$. There are injective maps

$$
M \stackrel{i_{1}}{\longrightarrow} M_{2} \stackrel{i_{2}}{\longrightarrow} M_{3}
$$

by which $m, i_{1}(m)$, and $i_{2} i_{1}(m)$ can be identified and hence the quotients $A / m$, $A_{2} / i_{1}(m)$, and $A_{3} / i_{2} i_{1}(m)$ compared. These happen to be either $A / m, C \times(A / m)$, or, $C$, where $C$ are the complex numbers. It is determined when each of the three alternatives occurs.

1. Multipliers of arbitrary rings. In this section the multiplier concept is developed in as general a setting as possible. All the subsequent definitions and results for a ring could just as well have been carried through for an algebra $A$ over a commutative ring $K$ provided all ideals, left ideals, subrings, and additive subgroups are assumed to be closed under multiplication from $K$. Note that $K$ always contains the integers. Thus the subsequent results which are derived only for rings, will later be used for algebras over the complex numbers.

1.1. Suppose $A$ and $S$ are associative rings where $A$ is a two sided $S$-module, i.e. $(T x) P=T(x P)$ for all $T, P \in S$ and $x \in A$. Assume that

$$
\text { (i) } x(T y)=(x T) y, \text { (ii) } T(x y)=(T x) y, \quad \text { (iii) }(x y) T=x(y T)
$$

holds for all $x, y \in A$ and $T \in S$. Then $\tilde{A}=S \times A$ becomes a ring under componentwise addition and under the following multiplication $(T, a)(P, b)=(T P, T b+a P+a b)$. Associativity can be readily verified. The ring $\tilde{A}$ contains $A=\{0\} \times A$ as an ideal (abbreviation: $A \triangleleft \tilde{A}$ ) and $S=S \times\{0\}$ as a subring with $S \cap A=\{0\}$ and $\tilde{A}=S+A$. Thus $\tilde{A}$ can be viewed either as $S \times A$ or as $S+A$, and it will be convenient sometimes to employ the one and sometimes the other interpretation.

1.2. Definition. A ring $\tilde{A}$ is said to be a splitting extension of $A$ by $S$ (or a semidirect product) if $\tilde{A}=S+A$, where $A \triangleleft \tilde{A}$, and where $S$ is a subring of $\tilde{A}$ with $S \cap A=\{0\}$.

Clearly, every splitting extension is of the form described in 1.1.

1.3. Definition. The multiplier $M(A)$ of any $\operatorname{ring} A$ is the set of all pairs $\left(T_{1}, T_{2}\right)$ of additive homomorphisms $T_{i}: A \rightarrow A$ such that

$$
\text { (i) } x\left(T_{1} y\right)=\left(T_{2} x\right) y, \quad \text { (ii) } T_{1}(x y)=\left(T_{1} x\right) y, \text { (iii) } T_{2}(x y)=x\left(T_{2} y\right)
$$

for all $x, y \in A$. Write $T=\left(T_{1}, T_{2}\right)$ and $T x=T_{1} x, x T=T_{2} x$. Left and right multiplications by an element $x \in A$ give maps $L_{x}, R_{x}: A \rightarrow A$ by $L_{x} a=x a, R_{x} a=a x$ 
for $a \in A$. Let $\mu: A \rightarrow M(A)$ and $\bar{x} \in M(A)$ for $x \in A$ be defined by $\mu(x)=\bar{x}=\left(L_{x}, R_{x}\right)$; write $\mu=\mu_{A}$ if the dependence on $A$ is important.

1.4. For any ring $A$, the multiplier $M(A)$ is a ring under the componentwise addition and the multiplication $T P=\left(T_{1} P_{1}, P_{2} T_{2}\right)$ where $T=\left(T_{1}, T_{2}\right), P=\left(P_{1}, P_{2}\right)$ $\in M(A)$. Let $T=\left(T_{1}, T_{2}\right) \in M(A)$ and $x \in A$. Then $\bar{x} \in M(A)$ and

$$
T \bar{x}=\left(T_{1} x\right)^{-}, \quad \bar{x} T=\left(T_{2} x\right)^{-} .
$$

Note that $T x=T_{1} x, x T=T_{2} x \in A$ while $T \bar{x}, \bar{x} T \in M(A)$. If $T x \neq 0$ belongs to the annihilator of $A$ in $A$, then $T \bar{x}=0$. Alternatively, $M(A)$ can be described as all $T$ such that $A$ is a two sided module satisfying 1.1 (i), (ii), and (iii). The map $\mu: A \rightarrow M(A)$ is a homomorphism whose kernel is the two sided annihilator of $A$ in $A$, i.e. ann $A=\{z \in A \mid z A=A z=0\}$. Thus $\bar{A}=\{\bar{x} \mid x \in A\} \cong A /$ ann $A$. Note that $M(A)$ has an identity, that $M(A)=A$ if $1 \in A$, and that $\bar{A} \triangleleft M(A)$.

Suppose $D$ is a ring with $\bar{A} \triangleleft D$ such that for any $\operatorname{ring} B$ with $A \triangleleft B$, there is a homomorphism $B \rightarrow D$ giving a commutative diagram

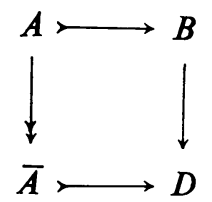

Then the ring $D=M(A)$ has this property and $M(A)$ is maximal with respect to this property. That is, if $\bar{A} \triangleleft D$ is essential (for any $\{0\} \neq J \triangleleft D, J \cap \bar{A} \neq\{0\}$ ), then $D$ and $M(A)$ are isomorphic under an isomorphism leaving $\bar{A}$ pointwise fixed. In particular, if ann $A=\{0\}$, and $A \triangleleft B$ is essential, then $A \subseteq B \subseteq M(A)$.

1.5. If the right and left annihilators of $A$ in $A$ are zero, i.e. if

$$
\{z \in A \mid z A=0\}=\{0\}, \quad\{z \in A \mid A z=0\}=\{0\},
$$

then conditions (ii) and (iii) in the Definition 1.3 of $M(A)$ are consequences of (i).

Proof. Since for any $x, y \in A$,

(ii) $z T_{1}(x y)=\left(T_{2} z\right) x y=z\left(T_{1} x\right) y$,

(iii) $T_{2}(x y) z=x y\left(T_{1} z\right)=x\left(T_{2} y\right) z$

holds for all $z \in A$, it follows that $T(x y)=(T x) y$ and $(x y) T=x(y T)$.

1.6. The following left ideals $L \subset A$ are left $M(A)$-ideals:

(i) $L=\{a-a u \mid a \in A\}$ for some $u \in A$;

(ii) $L=G: A=\{a \in A \mid A a \subseteq G\}$ where $G$ is any additive subgroup of $A$;

(iii) $L=A L$.

It would be interesting to find out under what conditions every regular maximal left ideal of a ring is of the form (i) in 1.6.

1.7. Let $f: A \rightarrow B$ be a surjective homomorphism of any rings $A, B$ whose kernel is $I$ and suppose that $\{z \in B \mid z B=0\}=\{z \in B \mid B z=0\}=\{0\}$. Define $M f: M(A) \rightarrow M(B)$ 
as follows. For $T \in M(A)$ and $b \in B$, choose any $a \in A$ with $f(a)=b$ and set $(M f)(T) b$ $=f(T a)$. Let $\bar{I} \subset M(A)$ be the ideal $\bar{I}=\{T \in M(A) \mid T A \cup A T \subseteq I\}$. Define $i: M(A) / \bar{I} \rightarrow$ $M(B)$ by $i(T+\bar{I}) b=f(T a)$ and $b i(T+\bar{I})=f(a T)$, where $T \in M(A)$ and where $a \in A$ is any element with $b=f(a)$.

(i) There is a commutative diagram

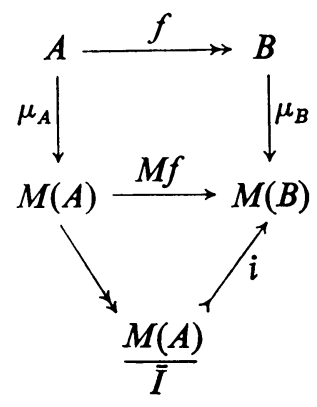

The kernel of $M f$ is $\bar{I}$; $i$ is given by the canonical epic-monic factorization of $M f$ and hence $i$ is monic.

(ii) $M$ is a functor from the category $\mathscr{B}$ of surjective ring homomorphisms of rings with zero left and zero right annihilators into the category of homomorphisms of rings with identity.

Proof. (i) and (ii). If two elements $a, a^{\prime} \in A$ satisfy $f(a)=f\left(a^{\prime}\right)=b$, then since

$$
\begin{aligned}
f(x) f(T a) & =f(x T) f(a), \\
f(x) f\left(T a^{\prime}\right) & =f(x T) f\left(a^{\prime}\right), \\
f(x) f\left(T a-T a^{\prime}\right) & =0
\end{aligned}
$$

holds for all $x \in A$, since $f(A)=B$, and since $\{z \in B \mid B z=0\}=\{0\}$, it follows that $f(T a)=f\left(T a^{\prime}\right)$ and that $M f(T)$ is well defined. If $T, P \in M(A)$, and $b=f(a) \in B$, then $M f(P T) b=f((P T) a)=M f(P)[f(T a)]=M f(P)[M f(T) b]$. Thus

$$
M f(P T)=M f(P) M f(T) .
$$

Linearity is clear and $M f$ is a homomorphism with $\bar{I} \subseteq$ kernel $M f$. Conversely, if $M f(T) B=B M f(T)=\{0\}$, then $f(T x)=f(x T)=0$ for all $x \in A$; thus $T A \cup A T \subseteq I$ and $T \in \bar{I}$. Hence $\bar{I}$ is the kernel of $M f$ and (i) and (ii) hold.

1.8. It is an open question whether the map $i$ in 1.7 is surjective in case $I$ is a primitive ideal. Consider a Hilbert space $H$ of Hilbert space dimension $\geqq \aleph_{1}$. Let $L H$ denote the bounded and $I$ the compact operators. Suppose $A$ is a closed proper ideal in $I \subset A \subset L H$ with $I \neq A$. The present author is unable even to answer the question for this particular $A$ and $I$.

One of the exercises in [9, p. 192, Exercise 11] suggests a method for constructing a complete topological group $G$ and a complete subgroup $H$ such that the quotient group $G / H$ is not complete. It is known that if $G$ has a countable dense subset, that 
then $G / H$ is complete. This construction from [9] has inspired the next example, which shows that even in the commutative case, the map $i$ and hence $M f$ need not be surjective.

1.9. EXAMPLE. Consider a topological space $T$. For any subset $Y \supseteq T, C^{b}(Y)$ denotes all bounded continuous complex valued functions on $Y ; C_{0}(Y) \subset C^{b}(Y)$ denotes those functions which tend to zero outside of compact subsets of $Y$; while $C_{E}(Y) \subset C^{b}(Y)$ is the subring of all those $f \in C^{b}(Y)$ which extend continuously to all of $T ; \quad Y^{\perp}$ is defined as $Y^{\perp}=\left\{f \in C^{b}(T)|f| Y=0\right\}$. Map $C^{b}(T) \rightarrow C_{E}(Y)$ by restriction $f \rightarrow f \mid Y$; the kernel is $Y^{\perp}$. Thus $C_{E}(Y) \cong C^{b}(T) / Y^{\perp}$. In the notation of 1.7, the restriction map $f \rightarrow f \mid Y$ gives a surjection $A=C_{0}(T) \rightarrow B=C_{0}(Y)$. In order for this map to be even defined, i.e. so that $f \mid Y \in C_{0}(Y)$, the set $Y$ has to be closed. (In particular, it is not possible to take $Y$ dense in $T$.) Thus the fundamental diagram for this case becomes

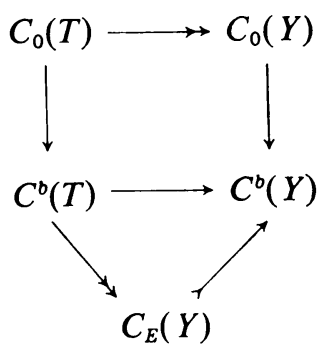

Consider Tychonoff's plank $T=[0, \Omega] \times[0, \omega] /\{(\Omega, \omega)\}$ in the usual order topology, where $\omega$ is the first infinite and $\Omega$ the first uncountable ordinal. Since any continuous function on $[0, \Omega]$ is eventually constant, for any continuous function on $T$ and each $0 \leqq n \leqq \omega$, there is an ordinal $\alpha(n)$ and a real number $r(n)$ such that $f((n, \beta))=r(n)$ for all $\beta \geqq \alpha(n)$. Set $\alpha=\sup \{\alpha(n) \mid 0 \leqq n \leqq \omega\}$. Then $\alpha \neq \Omega$, and $f$ is constant on each horizontal line segment of the rectangle $[\alpha, \Omega] \times[0, \omega] /\{(\Omega, \omega)\}$. Since $f$ is continuous, the $r(n)$ tend to $r(\omega)$. Let $Y$ be the closed subset $Y=\{\Omega\} \times[0, \omega)$ of $T$. Then $C_{E}(Y) \neq C^{b}(Y)$; for example the function $g((\Omega, n))=(-1)^{n}$ defined on $Y$ cannot be continuously extended to all of $T$. The sup-norm makes $C_{0}(T)$, $C_{0}(Y), C^{b}(T), C^{b}(Y)$, and $Y^{\perp}$ into $C^{*}$-algebras. Then the quotient norm on $C^{b}(T) / Y^{\perp}$ is the sup-norm on $C_{E}(Y)$. Another different topology may be put on these rings. They are additive topological groups in the topology of uniform convergence on compact subsets. Again the quotient topology on $C^{b}(T) / Y^{\perp} \cong C_{E}(Y)$ also in this case is the one of uniform convergence on compact subsets of $Y$. This second topology is properly smaller than the sup-norm for all the rings except $C_{0}(T)$ and $C_{0}(Y)$. Although both $C^{b}(T)$ and $Y^{\perp}$ are complete, $C_{E}(Y)$ is not complete.

The functor $M$ will be characterized as the left adjoint of a certain forgetful functor. In order to avoid a lengthy discussion, the terminology and some of the notation of [8, pp. 61-67] which by now is standard, will be used.

1.10. Suppose $\mathscr{A}$ and $\mathscr{B}$ are categories and $T: \mathscr{A} \rightarrow \mathscr{B}, S: \mathscr{B} \rightarrow \mathscr{A}$ are functors. 
Then $S$ is a left adjoint of $T$ if there is a natural equivalence of hom-functors $\phi: \mathscr{A}(S \cdot, \cdot) \rightarrow \mathscr{B}(\cdot, T \cdot)$. A necessary and sufficient condition for this is that there be a natural transformation $\rho: I \rightarrow T S$ of the identity functor $I$ on $\mathscr{B}$ such that for any morphism $y: B \rightarrow T A$ in $\mathscr{B}$, there exists a unique $x: S B \rightarrow A$ such that the following diagram commutes:

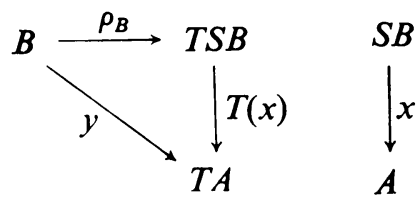

It should be noted that $x$ is unique only subject to choice of $\rho$.

1.11. Now specialize $\mathscr{B}$ as the category of surjective morphisms of rings with zero left and zero right annihilators. A category $\mathscr{A}$ will be defined. The objects (notation: $\mathrm{Ob} \mathscr{A})$ of the category $\mathscr{A}$ will be all the functions $\mu_{D}: D \rightarrow M(D)$ which map a ring $D$ into its multiplier. If $\mu_{D}, \mu_{E} \in \mathrm{Ob} \mathscr{A}$, then the maps of $\mathscr{A}$ (notation: Map $\mathscr{A}$ ) are pairs $(f, M f)$, where $f: D \rightarrow E$ is any surjective ring homomorphism, i.e. a morphism of $\mathscr{A}$ is a commutative diagram:

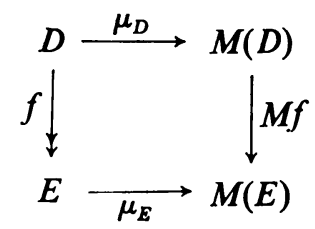

Let $S: \mathscr{B} \rightarrow \mathscr{A}$ be the functor which maps a morphism $f: D \rightarrow E$ of $\mathscr{B}$ into the morphism $(f, M f)$ of $\mathscr{A}$. If objects and identity maps are identified, then in particular, the value of $S$ at an object $D \in \mathrm{Ob} \mathscr{B}$ is $\mu_{D} \in \mathrm{Ob} \mathscr{A}$. Let $T: \mathscr{A} \rightarrow \mathscr{B}$ be the forgetful functor which sends the above $(f, M f) \in$ Map $\mathscr{A}$ into $f$. Note that $T$ maps $\mu_{D} \in \mathrm{Ob} \mathscr{A}$ into $D \in \mathrm{Ob} \mathscr{B}$.

Since $S$ is essentially the same as the functor $M$, a characterization of $S$ as in the next proposition is also a characterization of $M$.

1.12. Proposition. Suppose $T: \mathscr{A} \rightarrow \mathscr{B}$ and $S: \mathscr{B} \rightarrow \mathscr{A}$ are as in 1.11. Then $S$ is the left adjoint of the forgetful functor $T$.

Proof. The condition in 1.10 will be verified. Suppose $y: B \rightarrow T\left(\mu_{A}\right)$ in $\mathscr{B}$ is given, where $\mu_{A} \in \mathrm{Ob} \mathscr{A}$ and $T\left(\mu_{A}\right)=A$. Then $S B=\mu_{B} \in \mathrm{Ob} \mathscr{A}$. It will be shown that there exists a unique morphism $x \equiv(f, M f): S B \rightarrow \mu_{A}$ in $\mathscr{A}$ such that the following diagram commutes with $\rho$ as the identity on $\mathscr{B}$ :
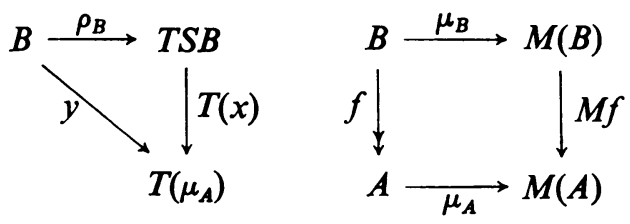
But $S B=\mu_{B}$ and $T S B=T\left(\mu_{B}\right)=B$, thus let $\rho$ be the identity. But $T(x)=f$ and $T\left(\mu_{A}\right)=A$. If $x$ is defined by setting $f=y$ and $x=(y, M y)$, then clearly the diagram commutes. There can be only one map $x$, because necessarily $T x=y$, and then $x$ is uniquely determined as $x=(y, M y)$.

The next observations are a partial attempt to determine all rings $A_{2}$ containing a given ring $A$ as a subring and having the same multiplier or centroid. Only those rings $A_{2}$ will be considered which contain $A$ as an essential subring, i.e. if $I \subset A_{2}$ is an ideal with $I \cap A=\{0\}$, then $I=\{0\}$.

1.13. Consider a ring $A$ with $A^{2}=A$, with ann $A=\{z \in A \mid z A=A z=0\}=\{0\}$, and an ideal $A_{2} \triangleleft M(A)$ with $A \subset A_{2} \subseteq M(A)$. Then $M\left(A_{2}\right)=M(A)$.

Proof. Clearly, ann $A_{2}=\{0\}$, because $A_{2} \subseteq M(A)$. Since $A_{2} \triangleleft M(A)$, and since ann $A=\{0\}$, it may be assumed that $A \subset A_{2} \subseteq M(A) \subseteq M\left(A_{2}\right)$. It suffices to show that $A \triangleleft M\left(A_{2}\right)$ and that $A \subset M\left(A_{2}\right)$ is essential. Note that $A^{2}=A$ and $A_{2} \subseteq M(A)$ implies that $A$ is automatically an ideal in any subring of $M(A)$ or $M\left(A_{2}\right)$ that contains $A$. Thus in particular $A \triangleleft A_{2}$ and also $A \triangleleft M\left(A_{2}\right)$. If $\{0\} \neq J \triangleleft M\left(A_{2}\right)$, then $J \cap A_{2} \neq\{0\}$. Then $J \cap A_{2} \triangleleft M\left(A_{2}\right)$. Since $A \triangleleft M(A)$ is essential and since $J \cap A_{2}$ $\triangleleft M(A), \quad\{0\} \neq A \cap\left(J \cap A_{2}\right)=A \cap J$. Thus $A \triangleleft M\left(A_{2}\right)$ is essential and hence $M\left(A_{2}\right) \subseteq M(A)$.

1.14. Let $A$ be any ring with $A^{2}=A$ and let $R$ be the centroid of $A$, i.e. $R=$ center $M(A)$. Suppose ann $A=\{0\}$ and that $A \subset A_{2} \subseteq R+A$, where $A_{2} \triangleleft R+A$. Then the centroid of $A_{2}$ is also $R$.

Proof. Let $R_{2}$ be the centroid of $A_{2}$. If $S=A_{2} \cap R$, then $A_{2}=S+A$ is an extension of $A$ where $S$ is a subring of $A_{2}$ with $S=$ center $A_{2}$ and with $S \triangleleft R$. Since $A_{2} \triangleleft R+A$, it follows that $R \subseteq R_{2}$. Thus $A \subset A_{2} \subseteq R+A \subseteq R_{2}+A$ and $A^{2}=A$ implies that $A$ is an ideal in $A_{2}, R+A$, and $R_{2}+A$. Furthermore, for any $\rho \in R_{2}, \rho$ (center $A_{2}$ ) $=\rho(S) \subseteq S$. The restriction $\rho \mid A: A \rightarrow A$ belongs to $R$. Suppose $\rho \neq \sigma \in R_{2}$ with $\rho|A=\sigma| A$. For $s \in S \subset A_{2}$, the elements $\rho(s), \sigma(s) \in S$. Since $\rho \neq \sigma$, there is an $s \in S$ at which $\rho(s) \neq \sigma(s)$. Since $S \subseteq R$, this means that there is an element $x \in A$ with $\rho(s) x \neq \sigma(s) x$. But then $\rho \in R_{2} \subset M\left(A_{2}\right), s, x \in A_{2}$ and thus $\rho(s) x=\rho(s x)=s(\rho x)$. Hence $s(\rho x)=s(\sigma x)=\sigma(s) x$ gives a contradiction.

2. Arbitrary extensions. The primitive ideal structure of a splitting extension $S+A$ of a $C^{*}$-algebra $A$ with $S \subset$ center $M(A)$ can be described rather thoroughly [4]. Thus it seems that the next logical step in developing the subject further would be to drop all the $C^{*}$-assumptions and determine the primitive ideal structure of an arbitrary extension $A \subset \hat{A}$ in terms of $A$ and $\hat{A} / A$. Unfortunately, at this level of generality the results are meager, and the little that can be said is contained in the next theorem and its corollaries. For us, the main application of the theorem will be in the case when $\hat{A}$ is a splitting extension, and even more important, for the case when $\hat{A}=M(A)$. However, in this section no assumptions are imposed on 
$A \subset \hat{A}$ other than that $A$ is an ideal in $\hat{A}$; it is not even assumed that $\hat{A}$ has identity.

Some notation and facts about primitive ideals are recalled in a form in which they will later be applied.

2.1. A simple $A$-module $V$ is a left module containing no submodules with $A V=V \neq\{0\}$. Suppose $A$ is any ring and $L$ is a regular maximal left ideal, i.e. with a right unit $u \in A$ such that $a-a u \in L$ for all $a \in A$. Then $q=L: A=\{a \in A \mid a A \subseteq L\}$ is primitive and $V=A-L=\{a+L \mid a \in A\}$ is simple. In particular, $A V=V \neq\{0\}$ in this case simply means that $A^{2}+L=A$. For any ring, Prim $A$ will denote the set of all primitive ideals of $A$. The next three observations apply to any quotient ideal $q$ of the form $q=L: A$ for some regular left ideal $L$. (To be more precise, (i) and (ii) only require that $L: A \subseteq L$, while (iii) holds for any $q=L: A$ for some left ideal $L$.)

(i) If $L$ is any regular left ideal, then $L: A$ is the unique biggest ideal of $A$ contained inside $L$. (Thus if every primitive ideal is contained in a unique regular maximal left ideal, then clearly the modular primitive ideals coincide with the maximal modular ideals. The converse fails even for a finite matrix ring.)

(ii) $q: A=L: A$. Clearly, $q: A \subseteq L: A$ since $q \subseteq L$ if $L$ is regular. Conversely, $L: A \equiv q \subseteq q: A$, since $q$ is a two-sided ideal.

(iii) If $Z=$ center $A$, then $L \cap Z \subseteq q$. For if $z \in L \cap Z$, then $z A=A z \subseteq A L \subseteq L$. If $q \subset L$, then $q \cap Z \subseteq L \cap Z$ and $L \cap Z=q \cap Z$.

2.2. Notation. Suppose $\hat{A}$ is any ring containing $A$ as an ideal. For any ideal $I \subset A$ define $\hat{I}=\{\alpha \in \hat{A} \mid \alpha A \subseteq I\}$. For any left ideal $L \subset A$, define $\hat{L}=\{\alpha \in \hat{A} \mid A \alpha \subseteq L\}$. Then $\hat{I}$ is a right and $\hat{L}$ a left ideal of $\hat{A}$. Elements of $A$ will be denoted by small Latin letters $a, x, y, \ldots$ while those of $\hat{A}$ by small Greek $\alpha, \beta, \gamma, \ldots$

In view of the usual embedding of Prim $A$ as a subset of Prim $\hat{A}$, it may be asked whether there is a similar correspondence between the associated left ideals. Among other things, the next Theorem I and its Corollary 1 completely answers this question.

B. E. Johnson showed [7] that for a ring with no left and also no right annihilators, there is a one to one correspondence between maximal modular left and maximal modular two sided ideals of $A$ and $M(A)$. The proofs below require no assumptions on the annihilators and replace $M(A)$ by an arbitrary ring $\hat{A}$ containing $A$ as an ideal.

2.3. TheOREM I. Let $\hat{A}$ be any ring and $A \triangleleft \hat{A}$. (It is not assumed that $\hat{A}$ has an identity.) Suppose $L \subset A$ is any regular maximal left ideal of $A$ with right unit $u \in A$ such that $a-a u \in L$ for $a \in A$. For $q \in \operatorname{Prim} A$ of the form $q=L: A=\{a \in A \mid a A \subseteq L\}$, define

$$
\hat{L}=\{\alpha \in \hat{A} \mid A \alpha \subseteq L\}, \quad \hat{q}=\{\alpha \in \hat{A} \mid \alpha A \subseteq q\} .
$$

Then the following conclusions hold.

(1) (a) $L$ is a left $\hat{A}$ ideal, i.e. $\hat{A} L \subseteq L$;

(b) $q$ is an ideal of $\hat{A}$;

(c) $\hat{q}$ is an ideal of $\hat{A}$. 
(2) $\hat{L}$ is a regular maximal left ideal in $\hat{A}$ with $u \in A$ a relative right identity for $\hat{L}$.

(3) $\hat{L} \cap A=L, \hat{q} \cap A=q$.

(4) $\hat{q}=\hat{L}: \hat{A}=\{\alpha \in \hat{A} \mid \alpha \hat{A} \subseteq \hat{L}\}$; in particular, $\hat{q} \in \operatorname{Prim} \hat{A}$.

(5) Any simple A-module $V$ becomes a simple $\hat{A}$-module under a unique natural action. Furthermore, if $q=\{a \in A \mid a V=0\}$, then $\hat{q}=\{\alpha \in \hat{A} \mid \alpha V=0\}$.

(6) The simple A-module $A-L=\{a+L \mid a \in A\}$ becomes an $\hat{A}$-module under

$$
\alpha(a+L)=\alpha a+L, \quad a \in A, \alpha \in \hat{A} .
$$

(7) The simple $\hat{A}$-module $\hat{A}-\hat{L}$ is $\hat{A}$-isomorphic to the $\hat{A}$-module $A-L$ under

$$
\hat{A}-\hat{L} \rightarrow A-L, \quad \alpha+\hat{L} \rightarrow \alpha u+L, \quad \alpha \in \hat{A} .
$$

Proof. (1) (a) By the maximality of $L$, if $\hat{A} L \nsubseteq L$, then $A=\hat{A} L+L$. Thus $A=$ $A^{2}+L=A(\hat{A} L+L)+L \subseteq L$ gives a contradiction and hence $\hat{A} L \subseteq L$. (1) (b) If $\alpha \in \hat{A}$ and $x \in q$, then $x A \subseteq L$ and $\alpha x A \subseteq \alpha L \subseteq \hat{A} L \subseteq L$. Also $x \alpha A \subseteq L$. Thus $q$ is actually an ideal in $\hat{A}$. (1) (c) Clearly, $\hat{q}$ is a right $\hat{A}$-ideal. Suppose $\alpha \in \hat{q}$, or $\alpha A \subseteq q$ and $\beta \in \hat{A}$ is arbitrary. Then $\beta \alpha \in \hat{q}$ provided for any $x \in A, \beta \alpha x \in q$ or $\beta \alpha x A \subseteq L$. Now $\alpha x \in q$ implies that $\alpha x A \subseteq L$ and $\beta \alpha x A \subseteq \beta L \subseteq L$, since $L$ has been shown to be a left $\hat{A}$-ideal. Thus $\beta \alpha x \in q$, or $\beta \alpha \in \hat{q}$, or $\hat{q}$ is an ideal in $\hat{A}$.

(2) Since for any $x \in A$ and $\alpha \in \hat{A}, x(\alpha-\alpha u)=(x \alpha)-(x \alpha) u \in L$, it follows from the definition of $\hat{L}$ that $\alpha-\alpha u \in \hat{L}$. Thus $u$ is also a relative right identity for $\hat{L}$. If $L_{1} \subset \hat{A}$ is a proper left ideal $L_{1}$ of $\hat{A}$ with $\hat{L} \subset L_{1}$ properly, then $A L_{1} \nsubseteq L$. Since $A L_{1}+L$ is a left ideal of $A$, by maximality of $L, A=A L_{1}+L$. Since $L \subset \hat{L} \subset L_{1}$, $u \in A L_{1}+L \subset L_{1}$. Since $L_{1}$ contains its relative identity, $L_{1}=\hat{A}$ which is a contradiction.

(3) Clearly $L \subseteq \hat{L} \cap A=\{a \in A \mid L a \subseteq L\}$. Since $A(A-L)=A-L$, for any $x \in A$ we have

$$
x \notin L \Leftrightarrow A x+L=A \Leftrightarrow A x \notin L \Leftrightarrow x \notin \hat{L} .
$$

Thus $\hat{L} \cap A \subseteq L$ and $L=\hat{L} \cap A$. It follows from the definition of $\hat{q}$ that

$$
\hat{q} \cap A=\{\alpha \in A \mid \alpha A \subseteq q\}=q .
$$

(4) To show that $\hat{q} \subseteq \hat{L}: \hat{A}$, take $\alpha \in \hat{q}=\{\alpha \mid \alpha A \subseteq q\}$. It has to be shown that $\alpha \hat{A} \subseteq \hat{L}=\{\beta \mid A \beta \subseteq L\}$, i.e. that for any $\gamma \in \hat{A}, \alpha \gamma \in \hat{L}$, or that $A \alpha \gamma \subseteq L$. Since $\gamma u-\gamma \in \hat{L}$, also $A \alpha(\gamma u-\gamma) \subseteq L$. Thus it suffices to show that $A \alpha \gamma u \subseteq L$. But $\alpha \in \hat{q}$ implies that $\alpha(\gamma u) \in q$ and hence that $A \alpha \gamma u \subseteq A q \subseteq q$. Thus $\hat{q} \subseteq \hat{L}: \hat{A}$.

To show the other inclusion that $\hat{L}: \hat{A} \subseteq \hat{q}$, take $\alpha \in \hat{L}: \hat{A}$, i.e. $\alpha \hat{A} \subseteq \hat{L}$. It has to be shown that $\alpha \in \hat{q}$, or $\alpha A \subseteq q$, or that $(\alpha A) A \subseteq L$. But for any $x \in A, \alpha(x A) \subseteq \alpha A$ $\subseteq \alpha \hat{A} \subseteq \hat{L}$ and $\alpha x A \subseteq \hat{L} \cap A=L$, or $\alpha x \in q$. Since $x$ was arbitrary, $\alpha A \subseteq q$. Thus $\alpha \in \hat{q}$ and $\hat{L}: \hat{A}=\hat{q} \in \operatorname{Prim} \hat{A}$.

(5) If $V$ is any simple $A$-module with $q=0: V=\{a \in A \mid a V=0\}$, then for any fixed $0 \neq v \in V, A v=V$. Set $L^{\prime}=0: v=\{a \in A \mid a v=0\}$. Since $L^{\prime}$ is a regular maximal left ideal of $A$, it follows from (1) that $\hat{A} L^{\prime} \subseteq L^{\prime}$. Define an action of $\hat{A}$ on $V$ as 
follows. For $w \in V$ and $\alpha \in \hat{A}$, write $w=a v$ for some $a \in A$ and define $\alpha w=\alpha a v$. If $a v=a_{1} v$ for some other $a_{1} \in A$, it has to be shown that $\alpha a v=\alpha a_{1} v$. Set $x=a-a_{1}$. Since $x v=0, x \in L^{\prime}$ and $\alpha x \in \alpha L^{\prime} \subseteq L^{\prime}$. If $\alpha V=0$, then since $V=A V, \alpha V=\alpha A V$, so $\alpha A \subseteq q$ or $\alpha \in \hat{q}$. Thus $\{\alpha \in A \mid \alpha V=0\}=\hat{q}$. The above definition of the action of $\hat{A}$ on $V$ does not depend on the choice of $0 \neq v \in V$. For if $0 \neq v_{1}, v_{2} \in V$ with $a_{1} v_{1}=a_{2} v_{2}$, then it will be shown that for all $\alpha \in \hat{A}$, we have $\alpha a_{1} v_{1}=\alpha a_{2} v_{2}$. With $0 \neq v \in V$ as above, map $A \rightarrow V$ by $a \rightarrow a v$ so that the kernel is $L^{\prime}=0: v$. Then $A-L^{\prime}$ and $V$ are isomorphic $A$-modules and $x_{i}+L^{\prime} \rightarrow v_{i}$ for some $x_{i} \notin L^{\prime}$ for $i=1,2$. Now $a_{1} v_{1}=a_{2} v_{2}$ implies that $a_{1} x_{1}+L^{\prime}=a_{2} x_{2}+L^{\prime}$ and $a_{1} x_{1}-a_{2} x_{2} \in L^{\prime}$. Since $\alpha L^{\prime} \subseteq L^{\prime}$, also $\alpha\left(a_{1} x_{1}-a_{2} x_{2}\right) \in L^{\prime}$, and thus $\alpha a_{1} v_{1}=\alpha a_{2} v_{2}$.

(6) Since for any element $a \in A, a u+L=a+L$, the preceding extension of the action of $\hat{A}$ to $V=A-L$ becomes $\alpha a u+L=\alpha a+L$.

(7) An element $\alpha \in \hat{A}$ belongs to $\hat{L}$ if and only if $\alpha u \in L$; for if $\alpha \in \hat{L}$, then $\alpha u=(\alpha u-\alpha)+\alpha \in \hat{L} \cap A=L$. Conversely, if $\alpha u \in L$, then since $\alpha-\alpha u \in \hat{L}$, also $\alpha=(\alpha-\alpha u)+\alpha u \in \mathcal{L}$. Thus the map

$$
\hat{A}-\hat{L} \rightarrow A-L, \quad \alpha+\hat{L} \rightarrow \alpha u+L
$$

is an isomorphism of $\hat{A}-\hat{L}$ onto $A-L$. If $\hat{A}$ acts on $A-L$ under the action given by (6), then this clearly is an $\hat{A}$-isomorphism.

It is well known (also see 2.3(3)), that there is a bijective map

$$
q \rightarrow \hat{q}: \operatorname{Prim} A \rightarrow\{J \in \operatorname{Prim} \hat{A} \mid A \ddagger J\}
$$

with inverse $J \rightarrow J \cap A$.

2.4. Corollary 1 to Theorem I. Under the hypotheses and in the notation of Theorem $\mathrm{I}$, let $q \in \operatorname{Prim} A$ be fixed and $\hat{q} \in \operatorname{Prim} \hat{A}$ be the unique primitive ideal with $\hat{q} \cap A=q$. Let $L(q)$ and $L(\hat{q})$ denote the set of regular maximal left ideals determining $q$ and $\hat{q}$ respectively. Then

(8) $L \rightarrow \hat{L}: L(q) \rightarrow L(\hat{q})$ is a bijection,

(9) whose inverse is $N \rightarrow N \cap A: L(\hat{q}) \rightarrow L(q)$.

Proof. By Theorem I, $L \rightarrow \hat{L}$ is well defined, since for $L \in L(q)$, also $\hat{L} \in L(\hat{q})$.

(a) Next it will be shown that for $N \in L(\hat{q}), N \cap A$ is a regular maximal left ideal of $A$. Since $A \nsubseteq \hat{q}$ and $\hat{q}=\{\alpha \in A \mid \alpha \hat{A} \subseteq N\}$ it follows that $A \hat{A}+N=\hat{A}$. If $\eta \in \hat{A}$ is a relative right identity for $N$ modulo $\hat{A}$, then $\eta=e+t$ for some $e \in A \hat{A}$ and $t \in N$. If $\alpha \in \hat{A}$ is any element, then $\alpha \eta-\alpha=\alpha e-\alpha+\alpha t$. Since $\alpha t \in N$, we have $\alpha e-\alpha \in N$. Thus $e \in A$ is a relative right identity for $\hat{A}$ modulo $N$, and consequently also for $A$ modulo $A \cap N$ as well. Now suppose $N \cap A \subset L_{1}, N \cap A \neq L_{1}$, where $L_{1}$ is a regular maximal left ideal of $A$. Then $L_{1} \ddagger N$, since $L_{1} \ddagger N \cap A$. Theorem I shows that $\hat{A} L_{1} \subseteq L_{1}$. Thus $L_{1}+N$, being a left ideal of $\hat{A}$ properly containing $N$, is actually $L_{1}+N=\hat{A}$. Then $e=x+n$ with $x \in L_{1}$ and $n \in N$. Since $n \in N \cap A \subseteq L_{1}$, $e \in L_{1}$. Since $L_{1}$ contains its relative right identity, $L_{1}=A$, a contradiction.

(b) Now we show that $N \cap A \in L(q)$. Since $\hat{q}=\{\alpha \in \hat{A} \mid \alpha \hat{A} \subseteq N\}$, and since by 
2.3(3), $\hat{q} \cap A=q$, it follows that $q=\hat{q} \cap A=\{a \in A \mid a A \subseteq N\}=\{a \in A \mid a A \subseteq N \cap A\}$ $=(N \cap A): A$. Thus $N \cap A \in L(q)$. Hence the maps in (8) and (9) are well defined.

(c) To complete the proof and show that they are bijections, it suffices to show that if $N \in L(\hat{q})$, that then $(N \cap A)^{\wedge}=N$. Since by definition

$$
(N \cap A)^{\wedge}=\{\beta \in \hat{A} \mid A \beta \subseteq N\},
$$

it follows that $N \subseteq(N \cap A)^{\wedge}$. However, since both $N$ and also $(N \cap A)^{\wedge}$ (see 2.3(2)) are maximal, $N=(N \cap A)^{\wedge}$.

2.5. For any $A \triangleleft \hat{A}$, there is a map of the ideals of $A$ into the ideals of $\hat{A}$ given by $I \rightarrow \tilde{I} \equiv\{\alpha \in \hat{A} \mid \alpha A \cup A \alpha \subseteq I\}$. Note that $I \subseteq \tilde{I}$. Define $\hat{I}=\{\alpha \in \hat{A} \mid \alpha A \subseteq I\}$, a right ideal of $A$. Then $I=I$ provided one of the following two conditions holds:

(i) $I=N: A=\{a \in A \mid a A \subseteq N\}$, where $N \subset A$ is any regular left ideal of $A$ with relative right unit $u \in A$.

(ii) $A \cap \hat{I}=I$.

(iii) In particular, for any $q \in \operatorname{Prim} A, \tilde{q}=\hat{q} \in \operatorname{Prim} \hat{A}$.

Proof. (i) Clearly always $\tilde{I} \subseteq I$. If $\alpha \in I$, then $\alpha \in \tilde{I}$ provided $A \alpha \subseteq I$, or $(A \alpha) A \subseteq N$. For any $x \in A, x \in N$ if and only if $x u \in N$. But $\alpha A u \subseteq \alpha A \subseteq I \subseteq N$ and hence $A \alpha A u \subseteq N$. Thus $\tilde{I}=$ I.

(ii) First, $A \hat{I} \subseteq \hat{I}$, since $A(\hat{I} A) \subseteq A(I) \subseteq I$. Thus $A \hat{I} \subseteq \hat{I} \cap A=I$ and again $\tilde{I}=\hat{I}$.

(iii) Either (i) or (ii) implies (iii).

The last theorem and the last observation are now specialized to the case when $\hat{A}=M(A)$.

2.6. Corollary 2 to Theorem I. If $A$ is any ring and $V$ is any simple $A$-module, then

(i) $V$ is also a simple $M(A)$-module.

(ii) If $\tilde{A}=S+A$ with $A \triangleleft \tilde{A}$ and $S$ a subring, then there is a homomorphism $S \rightarrow M(A)$. Thus $V$ is an $S$ and an $\tilde{A}$-module.

Use of 2.3(1)(c) gives immediately a known result (see B. E. Johnson [7]).

2.7. Corollary 3 to Theorem I. Assume $A$ is any ring with

$$
\text { ann } A=\{z \in A \mid z A=A z=0\}=\{0\}
$$

and view $A$ as an ideal in $A \subset M(A)$.

(i) Each $q \in \operatorname{Prim} A$ is an ideal of $M(A)$.

(ii) The standard embedding of $\operatorname{Prim} A \rightarrow \operatorname{Prim} M(A)$ is given by

$$
q \rightarrow \tilde{q}=\{T \in M(A) \mid T A \subseteq q\}=\{T \in M(A) \mid T A \cup A T \subseteq q\} .
$$

The next corollary gives a method which could conceivably be used for computing $M(A)$ for primitive rings $A$.

2.8. Corollary 4 to Theorem I. Consider a primitive ring $A$ without an identity and a simple A-module $V$ with $0: V=\{a \in A \mid a V=0\}=\{0\}$. Let $D$ be the skew-field 
of all endomorphisms of $V$ which commute with $A$. Let $E(V)$ be the ring of all D-linear maps of $V$ into $V$ and view $A \subset E(V)$. Then $M(A)$ consists of all $T \in E(V)$ such that $T A \cup A T \subseteq A$.

Proof. All $T \in E(V)$ with the above property clearly belong to $M(A)$. Now $V$ is an $M(A)$-module. If $T \in M(A), d \in D$, and $w \in V$, then pick $0 \neq v \in V$ and write $w=a v$ for some $a \in A$. Then $T d a v=(T a)(d v)=d(T a) v$. Thus $T d=d T$ and $T \in E(V)$ with $T A \cup A T \subseteq A$.

3. Splitting extensions. The multiplier concept is particularly well-suited for dealing with splitting extensions $\tilde{A}=S \times A$ of a $\operatorname{ring} A$ where $S$ acts faithfully on $A$. The case when $S \subseteq R$, the centroid of $A$, is a special instance which was treated in [4]. Some portions of [4] can be generalized to apply in this more general case and these will not be considered here in detail.

3.1. If $\tilde{A}=S+A$ is any ring with $A \triangleleft \tilde{A}$ and where $S$ is a subring of $\tilde{A}$, then there is a homomorphism $S \rightarrow M(A)$, defined by $T \rightarrow\left(T_{1}, T_{2}\right)$, where $T_{1} x \equiv T x, T_{2} x \equiv x T$ for $T \in S$ and all $x \in A$. The kernel of this homomorphism is $\{T \in S \mid T A \cup A T=0\}$. Of course a ring of the form $S+A$ is interesting only if $S \cap A$ is known. However, if $S \times A$ is the semidirect product of $S$ and $A$ as in 1.2 , and if

$$
D=\{(s,-a) \mid s=a \in S \cap A\},
$$

then $\tilde{A} \cong(S \times A) / D$. Then $D$ belongs to the two sided annihilator of $\{0\} \times A$ in $S \times A$. Furthermore, if $S \subseteq M(A)$ and ann $A=\{z \in A \mid z A=A z=0\}=\{0\}$, then $D$ is exactly the annihilator of $A$.

3.2. Definition. Consider a completely arbitrary extension $A \triangleleft \tilde{A}$ of any ring $A$ ( $1 \in \tilde{A}$ is not assumed) and express $\tilde{A}=S+A$, where $S$ is some subring of $\tilde{A}$ (no assumptions on $S \cap A$ ). An ideal of the form $S_{1}+A_{1} \triangleleft \tilde{A}$ with

(a) $A_{1} \triangleleft \tilde{A}, \quad S_{1} \triangleleft S$

will be called a box ideal and a splitting box ideal (with respect to $S$ ) provided also

(b) $\left(S+A_{1}\right) \cap\left(S_{1}+A\right)=S_{1}+A_{1}$,

(c) $\left(S_{1}+A_{1}\right) \cap A=A_{1}$,

(d) $S \cap\left(S_{1}+A_{1}\right)=S_{1}$.

It follows as a consequence of (c) and (a) respectively that

(e) $S_{1} A \cup A S_{1} \subseteq A_{1}$,

(f) $S A_{1} \cup A_{1} S \subseteq A_{1}$

3.3. Consider a splitting box ideal $S_{1}+A_{1}$ in an extension $S+A$ of $A$ of the form 3.2 and define $\bar{A}=A / A_{1}, \bar{S}=S / S_{1}$. Then

(i) $\bar{S} \times \bar{A}$ is a splitting extension where for $\bar{T}=T+S_{1} \in \bar{S}$ and $\bar{a}=a+A_{1} \in \bar{A}$, $\bar{T} \bar{a} \equiv(T a)^{-}$and $\bar{a} \bar{T} \equiv(a T)^{-}$;

(ii) there is an isomorphism

$$
(\bar{T}, \bar{a}) \rightarrow T+a+\left(S_{1}+A_{1}\right): \bar{S} \times \bar{A} \rightarrow(S+A) /\left(S_{1}+A_{1}\right) .
$$


Proof. Conclusion (i) follows from 3.2(e) and (f). The map in (ii) is clearly a homomorphism. If $(\bar{T}, \bar{a})$ is in the kernel, then $T+a=T_{1}+a_{1} \in S_{1}+A_{1}$ with $T_{1} \in S_{1}, a_{1} \in A_{1}$. Then by $3.2(\mathrm{~b})$ we get

$$
T-a_{1}=T_{1}-a \in\left(S+A_{1}\right) \cap\left(S_{1}+A\right)=S_{1}+A_{1} .
$$

Then 3.2(c) and (d) show that

$$
a \in\left(S_{1}+A_{1}\right) \cap A=A_{1}, \quad T \in S \cap\left(S_{1}+A_{1}\right)=S_{1},
$$

and hence that $(\bar{T}, \bar{a})=(\overline{0}, \overline{0})$.

3.4. Remarks. 1. The isomorphism in 3.3(ii) can also be obtained indirectly in another way that brings out the significance of conditions 3.2 more clearly. If $J=S_{1}+A_{1}$, then

$$
\frac{S+A}{J}=\frac{S+J}{J}+\frac{A+J}{J}
$$

is an internal semidirect product because $(A+J) / J \triangleleft \tilde{A} / J$, where $(S+J) / J$ by $3.2(\mathrm{~b})$ is a subring with $(S+J) \cap(A+J)=J$. Furthermore 3.2(d) and (c) show that

$$
\frac{S+J}{J} \cong \frac{S}{S \cap J}=\frac{S}{S_{1}}, \quad \frac{A+J}{J} \cong \frac{A}{A \cap J}=\frac{A}{A_{1}}
$$

As abelian groups, $\tilde{A} / J \cong \bar{S} \times \bar{A}$. By simply transferring the multiplication from $\tilde{A} / J$ to the abelian group $\bar{S} \times \bar{A}$, both 3.3(i) and (ii) follow.

2. In 3.3, the two sided annihilator of $\bar{A}$ in $\bar{S}$ is $\left\{\bar{T} \in \bar{S} \mid T \in S, T A \cup A T \subseteq A_{1}\right\}$. In particular, $\bar{S}$ acts faithfully on $\bar{A}$ if $S_{1}=\left\{T \in S \mid T A \cup A T \subseteq A_{1}\right\}$.

3. In the previous remark, a second algebra $(S+A) / A_{1} \cong S \times\left(A / A_{1}\right)=S \times \bar{A}$ may be formed; $S_{1} \triangleleft S \times \bar{A}$ and $S_{1} \bar{A}=\bar{A} S_{1}=\{0\}$. Thus even when originally $S$ acted faithfully on $A$, now $S$ no longer acts faithfully on $\bar{A}$. For this reason it is not sufficient to consider only splitting extensions of the form $S \times A$ where $S \subset M(A)$ and $S$ acts faithfully on $A$.

All ideals of a splitting extension $S \times A$ will be identified. If $\tilde{A}=S+A$ is the more general kind of extension as in 3.1 with $\tilde{A} \cong(S \times A) / D$, then this will also serve to determine all the ideals of $\tilde{A}$.

3.5. Suppose $S \times A$ is any splitting extension and let $\pi: S \times A \rightarrow S$ be the natural projection. Suppose $S_{1}, \varphi, A_{1}$ is a triple where $S_{1} \triangleleft S$ is an ideal in $S, A_{1} \triangleleft A$ is an ideal in all of $S \times A$, and $\varphi: S_{1} \rightarrow A / A_{1}$ is a homomorphism satisfying the following for all $r \in S_{1}, s \in S$, and $a \in A$ :

(1) $\varphi(s r)=s \varphi(r), \quad \varphi(r s)=\varphi(r) s$,

(2) $\varphi(r)\left[a+A_{1}\right]=r a+A_{1}, \quad\left[a+A_{1}\right] \varphi(r)=a r+A_{1}$.

If $I$ is defined to be $I=\left\{(r,-c) \mid r \in S_{1}, c \in \varphi(r)\right\}$, then

(i) $I \subset S \times A$ is an ideal.

(ii) $\pi(I)=S_{1}, \quad I \cap A=A_{1}$. 
(iii) $\pi(I)=S_{1} \triangleleft S \times A \Leftrightarrow S_{1} A \cup A S_{1}=\{0\}$; since $S_{1} A \cup A S_{1} \subseteq A_{1}$ (see 3.2(f)), in particular, $A_{1}=\{0\}$ implies that $\pi(I) \triangleleft S \times A$.

(iv) The kernel of $\varphi$ is an ideal in $S \times A$ if $A_{1}=\{0\}$.

(v) If $\pi(I)=S$, and if $1 \in S$ acts as the identity on $A$, then pick an $e \in A$ such that $\varphi(1)=e+A \cap I \in A / A \cap I$. Then $\varphi(1)$ is the identity of $A / A \cap I$, then $I=S(1-e)$ $+I \cap A$, and $S \times A=I+A$ (where $A=\{0\} \times A, S=S \times\{0\}$ ).

Conversely, every ideal $I$ of $S \times A$ is of this form. Given $I$, define $A_{1}=I \cap A$, $S_{1}=\pi(I)$, and $\varphi: \pi(I) \rightarrow A / A \cap I$ by $\varphi(r)=a+A \cap I$ if $(r,-a) \in I$, where $r \in \pi(I)$ and $a \in A$.

Proof. Given $\varphi$, a trivial computation shows that the above defined $I$ is an ideal. The proof of the converse is omitted because the same techniques that have been used to prove the analogous result for $S \subseteq R=$ center $M(A)$, may also be used here [4, Proposition 1.5].

The next corollary will be needed later.

3.6. Corollary. Consider an extension $A \triangleleft \tilde{A}=S+A$ as in 3.1 and an ideal $T \triangleleft \tilde{A}$. Then $T$ can be of the following forms:

(i) $(T+A) \cap S \subseteq T \Rightarrow T=T \cap S+T \cap A$.

(ii) If $1 \in S$ acts as the identity on $A$ and if $(T+A) \cap S=S$, then there exists $e \in A, \quad 1=e+T \cap A \in A / T \cap A$ such that $T=S(1-e)+T \cap A$.

Proof. Conclusion (i) is trivial. (ii) Set $D=\{(a,-a) \in S \times A \mid a \in S \cap A\} \triangleleft S \times A$. Then $f_{1}$ is an isomorphism

$$
\begin{gathered}
S \times A \stackrel{f_{2}}{\longrightarrow} \frac{S \times A}{D} \stackrel{f_{1}}{\longrightarrow} S+A \\
(s, a) \longrightarrow(s, a)+D \longrightarrow s+a .
\end{gathered}
$$

Define $J$ and $I$ by

$$
J \equiv f_{1}^{-1}(T) \triangleleft \frac{S \times A}{D}, \quad I \equiv f_{2}^{-1}(J) \triangleleft S \times A .
$$

A routine computation shows that $I$ satisfies $3.5(\mathrm{v})$, and consequently that $f_{1} f_{2} I=T=S(1-e)+T \cap A$ is of the above form.

Now it will be convenient to utilize the notation of Theorem I.

3.7. Consider any extension $\hat{A}=S+A$ of the form 3.1, where $A$ is an ideal in $\hat{A}$ and $S$ is a subring and a primitive ideal $q \subset A$. Form $\hat{q}=\{\alpha \in \hat{A} \mid \alpha A \subseteq q\}$, and $\bar{q}=\{T \in S \mid T A \subseteq q\} \triangleleft S$. Assume $S \cap A=\{0\}$ and without loss of generality also that $S \subseteq M(A)$ and $\tilde{q}=\{\mathrm{T} \in M(A) \mid T A \subseteq q\}$.

(i) There are monomorphisms $\mu: A / q \rightarrow M(A / q)$ and $\lambda: S / \bar{q} \rightarrow M(A / q)$.

(ii) $\mu(A / q) \cap \lambda(S / \bar{q})=\{0\} \Leftrightarrow \hat{q}=\bar{q}+q$. 
Proof. (i) By 3.1 and 1.7, there is a commutative diagram

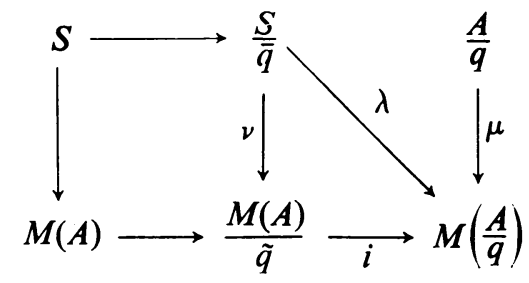

Obviously, $\lambda=i \circ \nu$ and $\lambda$ is given by

$$
\lambda(T+\bar{q})[a+q]=T a+q, \quad[a+q] \lambda(T+\bar{q})=a T+q \quad T \in S, a \in A .
$$

Since $A / q$ is isomorphic to a dense ring of linear transformations, ann $(A / q)=\{0\}$ and $\mu$ is monic. Now kernel $\lambda=\{T+\bar{q} \in S / \bar{q} \mid T A \cup A T \subseteq q\} \subseteq\{\bar{q}\}$. But $T A \subseteq q$ implies also that $A T \subseteq q$ by 2.7(ii). Thus kernel $\nu=\bar{q}$ and also $\nu$ is monic. I.e., $A / q$ and $S / \bar{q}$ may be viewed as an ideal and a subring of $M(A / q)$.

(ii) Clearly, $\bar{q}+q \subseteq \hat{q}$. By definition, $\hat{q}=\{T-c \mid T \in S, c \in A,(T-c) A \subseteq q\}$. However, for $T-c \in \hat{q}$, also $A(T-c) \subseteq A \hat{q} \subseteq A \cap \hat{q}=q$. Thus

$$
T-c \in \hat{q} \Leftrightarrow(T-c) A \cup A(T-c) \subseteq q \Leftrightarrow \mu(c+q)=\lambda(T+\bar{q}) \in \mu(A / q) \cap \lambda(S / \bar{q}) .
$$

But then $\hat{q}=\{T-c \mid \mu(c+q)=\lambda(T+\bar{q}) \in \mu(A / q) \cap \lambda(S / \bar{q})\}$. If $\mu(A / q) \cap \lambda(S / \bar{q})=\{0\}$ then $T-c \in \hat{q}$ only if $T \in \bar{q}$ and $c \in q$, so that $\hat{q}=\bar{q}+q$. If, on the other hand, $\hat{q}=\bar{q}+q$, and if $\mu(c+q)=\lambda(T+q) \in \mu(A / q) \cap \lambda(S / \bar{q})$, then $T-c \in \hat{q}$ and hence is of the form $T-c=T_{1}-c_{1}$ with $T_{1} \in \bar{q}, c_{1} \in q$. But then $T-T_{1}=c-c_{1} \in S \cap A=\{0\}$, so that $\mu(c+q)=\lambda(T+q)=0$.

4. The primitive ideal space of the multiplier. Since at the present time the main application of multipliers is for $C^{*}$-algebras, from now on $A$ will be a $C^{*}$ algebra. The results of [4] will be freely used in the remaining sections.

4.1. Notation. First, those algebras which will be later considered and their primitive ideal spaces are described. Consider an arbitrary $C^{*}$-algebra $A$ with or without an identity, with primitive ideal space Prim $A=B$, with centroid $R$, and $Z=R \cap A$ the center of $A$. Set $P=\operatorname{Prim} M(A)$, where $M(A)$ is the multiplier algebra of $A$. There is a map of ideals of $A$ into ideals of $M(A)$ given by

$$
I \rightarrow \tilde{I}=\{T \in M(A) \mid T A \cup A T \subseteq I\} .
$$

Thus $B \cong \widetilde{B}=\{\tilde{b} \mid b \in B\} \subset P$ is the usual embedding of $B$ as a hull-kernel dense open subset of $P$ (see 2.7). If $Y$ is the maximal ideal space of $R$, let $F: B \rightarrow Y$ be the map $F(b)=\tilde{b} \cap R=\{r \in R \mid r A \subseteq b\}$. Since $Z \subset R$ is an ideal, the maximal ideal space $Z(Y)$ of $Z$ may be embedded as an open subset $Z(Y) \subset Y$. If $C \times A$ is the splitting extension obtained by adjoining an identity to $A$ in the usual way, set $B^{\prime}=$ Prim $(C \times A)$ and let $\pi_{1}, \pi_{2}$ be the projections $\pi_{1}: B^{\prime} \times Y \rightarrow B^{\prime}$ and $\pi_{2}: B^{\prime} \times Y \rightarrow Y$. 
Our interest will be focused on four rings $A \equiv A_{1} \subseteq A_{2} \subseteq A_{3} \equiv R+A \subset M(A)$, where $A_{2}$ is any closed ideal in $R+A$, but $A_{2}$ is not necessarily an ideal in $M(A)$. As previously, $A_{2} \triangleleft A_{3}$ means that $A_{2}$ is a (not necessarily closed) ideal in $A_{3}$, although we will never encounter a nonclosed ideal. If $1 \in A$, then $A=A_{2}=R+A$ $=M(A), R=Z$, and all our statements about these algebras become trivial. Although $A_{2}$ will not be used until $\S 5$, it will be much more economical to define the objects associated with the four algebras all at once. Furthermore, a single statement about $A_{2}$ gives two others by specializing $A_{2}=A$ or $A_{2}=R+A$. Set $B_{3}=\operatorname{Prim} A_{3}$. As usual there are hull-kernel open subsets $B_{1} \subset B_{2} \subset B_{3}$ with $B_{i}=\left\{b_{3} \mid A_{i} \ddagger b_{3} \in B_{3}\right\}$, and $B_{i} \cong$ Prim $A_{i}=\left\{b_{i} \cap A_{i} \mid b_{i} \in B_{i}\right\}$. The following table may be helpful.

\begin{tabular}{l|l|l}
\multicolumn{1}{c|}{ algebra } & \multicolumn{1}{c|}{$\begin{array}{c}\text { primitive } \\
\text { ideal space }\end{array}$} & \multicolumn{1}{c}{ embedding } \\
\hline$A=A_{1}$ & $B_{1} \cong B \cong \widetilde{B}$ & $B_{1} \subset B_{3}, \widetilde{B} \subset P$ \\
$A_{2}$ & $B_{2}$ & $B_{2} \subset B_{3}$ \\
$A_{3}=R+A$ & $B_{3}$ & \\
$M(A)$ & $P$ & \\
$R=\operatorname{center} M(A)$ & $Y$ & $Z(Y) \subset Y$ \\
$Z=\operatorname{center} A$ & $Z(Y)$ &
\end{tabular}

It is necessary to isolate a few facts from [4, $\S 3.1$ and 3.3].

4.2. For any $C^{*}$-algebra $A$ whatever with or without an identity, there is a set of ideals $M$ and a map $\varphi: B \rightarrow M$ where

$$
b \in B, \quad p \equiv F(b), \quad m=m(p)=\varphi(b)=\bigcap\{b \in B \mid F(b)=p\} .
$$

By the adjoint-functor theorem or otherwise, there is the complete regularization $\varphi^{\prime}: B \rightarrow T$ in its hull-kernel topology so that any map of $B$ into a completely regular space factors uniquely through $\varphi^{\prime}$. If for $t \in T, \tau(t)$ is defined as

$$
\tau(t)=\bigcap\left\{b \in B \mid \varphi^{\prime}(b)=t\right\},
$$

then $\tau: T \rightarrow M$ is a bijection and $\varphi=\tau \circ \varphi^{\prime}$. (Alternatively, $M$ is the canonical image of $B$ in the Stone-Čech compactification of $B$.)

Some known or easily derivable useful properties of the maps $\varphi$ and $F$ are listed. If $b, q \in B$, if $p=F(b)$, and $m=m(p)=\varphi(b)$, then:

(i) $F(b)=F(q) \Leftrightarrow \varphi(b)=\varphi(q)$.

(ii) There is a bijective map of the subset $F(B) \subset Y$ onto $M$ given by

$$
F(B) \rightarrow M, \quad p \rightarrow m(p)=\bigcap\{q \in B \mid F(q)=p\} .
$$

(iii) $R \cap b=R \cap m=Z \cap b=Z \cap m=Z \cap F(b)$; furthermore

$$
F(b)=\{r \in R \mid r A \subseteq m\} \text {. }
$$

(iv) If $1 \in A$, then $F(b)=Z \cap b$ and $\varphi(b)=\bigcap\{q \in B \mid q \cap Z=b \cap Z\}$. 
4.3. If $A$ is any $C^{*}$-algebra $(1 \in A$ or $1 \notin A)$ with center $Z$ and $\varphi: B \rightarrow M$ as above, then for $b \in B=\operatorname{Prim} A$, conditions (i)-(iii) are equivalent:

(i) $Z \subseteq b$;

(ii) $Z \subseteq F(b)$;

(iii) $Z \subseteq \varphi(b)$.

Proof. First note that since $b=L: A$ for some regular maximal left ideal $L$ of $A$, it follows that $A \cap F(b)=Z \cap b$. (i) $\Rightarrow$ (ii). If $z \in Z \cap b$, then $z \in R, z A \subseteq b$, and hence $z \in F(b)$. (ii) $\Rightarrow$ (i). Since $Z \subseteq F(b)$, it follows that $Z \subseteq F(b) \cap A=Z \cap b \subseteq b$. (ii) $\Rightarrow$ (iii). Since $\varphi(b)=\bigcap\{q \in B \mid F(q)=F(b)\}$, and since $Z \subseteq F(q) \cap A \subseteq q$ for each $q$ in the intersection, also $Z \subseteq \varphi(b)$. Conclusion (iii) $\Rightarrow$ (i) is trivial, since $\varphi(b) \subseteq b$ implies $Z \subseteq b$.

4.4. For $A \subset A_{2} \triangleleft R+A$ as in 4.1, $R$ is also the centroid of $A_{2}$ by 1.14. If $S$ is defined as $S=A_{2} \cap R$, then $A_{2} \cap S=$ center $A_{2}$ and $A_{2}=S+A$. Since $F$ is defined in 4.1 for any $C^{*}$-algebra, there is also such a map $F_{2}: B_{2} \rightarrow Y$ for $A_{2}$ :

$$
\begin{aligned}
& B_{2} \longrightarrow \operatorname{Prim} A_{2} \longrightarrow Y, \\
& b_{2} \longrightarrow b_{2} \cap A_{2} \longrightarrow F_{2}\left(b_{2}\right)=\left\{r \in R \mid r A_{2} \subseteq b_{2} \cap A_{2}\right\} \in Y .
\end{aligned}
$$

Thus $F_{2}\left(b_{2}\right)=\left\{r \in R \mid r A_{2} \subseteq b_{2}\right\} \subseteq b_{2}$ and $F\left(b_{2}\right)=b_{2} \cap R$. The above holds in particular for $A_{2}=A$ or $A_{2}=R+A$. Thus there are maps as follows:

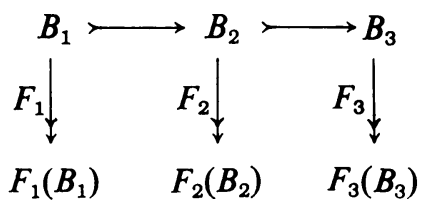

A distinct advantage of taking $B_{i} \subset B_{3}$ is that for $b_{i} \in B_{i}, F_{i}\left(b_{i}\right)=b_{i} \cap R$.

4.5. Taking the map $\varphi$ as in 4.2 separately for $A, A_{2}, A_{3}$ and identifying only here Prim $A \leftrightarrow B_{1}$, Prim $A_{2} \leftrightarrow B_{2}$, and Prim $A_{3} \leftrightarrow B_{3}$, and using 4.4 we obtain maps

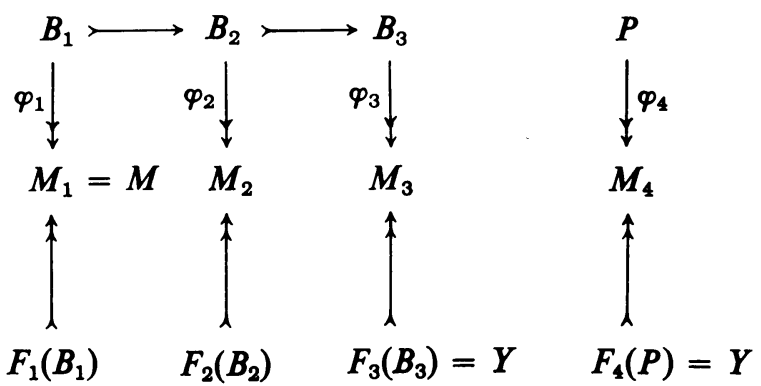


For $b_{i} \in B_{i}$, and $p=F_{i}\left(b_{i}\right) \in Y$ write $\varphi_{i}\left(b_{i}\right)=m_{i}=m_{i}(p)$. It should be stressed that although $B_{1}, B_{2}$, and $B_{3}$ consist of ideals of $A_{3}$, nevertheless $M_{1}, M_{2}$, and $M_{3}$ consist of ideals of $A, A_{2}$, and $A_{3}$ respectively. Also, $\varphi: B \rightarrow M=M_{1}$ differs from $\varphi_{1}: B_{1} \rightarrow M_{1}$ only in that $B$ has been replaced by $B_{1}$.

4.6. Lemma. If $i: M \rightarrow M_{3}$ is defined by $i(m(p))=m_{3}(p)$, then:

(i) $i(m)=\varphi_{3}\left(b_{1}\right)$ for any $b_{1} \in B_{1}$ with $m=\varphi_{1}\left(b_{1}\right)$; thus there is a commutative diagram

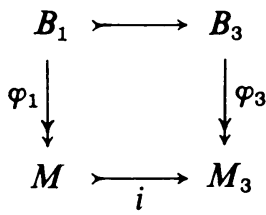

(ii) $i$ is an embedding;

(iii) $i(M) \subset M_{3}$ is a dense subset.

Proof. (i) Since center $A_{3}=R, \varphi_{3}\left(b_{1}\right)=\bigcap\left\{b_{3} \mid b_{3} \cap R=b_{1} \cap R\right\}$. If $q \in B_{1} \subset B_{3}$ with $\varphi_{1}\left(b_{1}\right)=\varphi_{1}(q)$, it suffices to show that $\varphi_{3}\left(b_{1}\right)=\varphi_{3}(q)$. However, $\varphi_{1}\left(b_{1}\right)=\varphi_{1}(q)$ implies that $F_{1}\left(b_{1}\right)=F_{1}(q)$. Thus $F_{1}\left(b_{1}\right)=b_{1} \cap R$ and $F_{1}(q)=q \cap R$, and hence $\varphi_{3}\left(b_{1}\right)=\varphi_{3}(q)$.

(ii) and (iii). It follows from [4, 3.16(2), (3), p. 194] that $i$ is an embedding. Since $B_{1}$ is dense in $B_{3}$, so is also $M$ in $M_{3}$.

REMARK. That the restriction $\varphi_{3} \mid\left(B_{3} \mid B_{1}\right)$ is a homeomorphism of compact Hausdorff spaces has been observed in [5].

In [4], Prim $(R+A)$ was computed. In the next paragraph these results are now utilized to concretely identify every primitive ideal of $A_{2}$.

4.7. Write $B=\mathscr{M} \cup \mathscr{N}$, where $\mathscr{M}$ are the modular and $\mathscr{N}$ the nonmodular ideals of $B$. There is a one-to-one correspondence between $B_{1}$ and the graph of $F$, graph $F=\{\langle b, F(b)\rangle \mid b \in B\}$ given by

$$
\begin{array}{ll}
b \in \mathscr{M}, e+b=1 \in A / b: & \langle b, F(b)\rangle=R(1-e)+b ; \\
b \in \mathscr{N}: & \langle b, F(b)\rangle=b+F(b) .
\end{array}
$$

Since the center of a primitive Banach algebra is either $\{0\}$ or $C$, and since $(Z+b) / b \subseteq$ center $A / b$, it follows that for $b \in \mathscr{N}$, we have $Z \subseteq b$.

Define $S$ by $S=A_{2} \cap R$ so that $A_{2}=S+A$ with $S \cap A \subseteq Z$ and $S \triangleleft R$. If $S \times A$ is the semidirect product (see 1.2) and $D \subset S \times A$ is the ideal of $S \times A$ which annihilates $A$, i.e. $D=\{(z,-z) \mid z \in Z\}$, then $S+A \cong(S \times A) / D$. Since for $b \in B$, $A \cap\langle b, F(b)\rangle=b \neq A$, it follows that also $A_{2} \ddagger\langle b, F(b)\rangle$. Thus all the spaces $B_{1} \subset B_{2} \subset B_{3}$ can be identified as subsets of graph $F \cup\{\langle A, p\rangle \mid p \in Y\}$ as follows:

$$
\begin{aligned}
& B_{1}=\operatorname{graph} F, \\
& B_{2}=B_{1} \cup\{\langle A, p\rangle \mid Z \subseteq p \in Y, S \nsubseteq p\}, \\
& B_{3}=B_{1} \cup\{\langle A, p\rangle \mid Z \subseteq p \in Y\} .
\end{aligned}
$$


Set $Y_{3}=\{p \in Y \mid Z \subseteq p\}$, and $Y_{2}=\left\{p \in Y_{3} \mid Z \subseteq p, S \nsubseteq p\right\}$. Thus

consists of

$$
\text { Prim } A_{2}=\left\{b_{2} \cap A_{2} \mid b_{2} \in B_{2}\right\}
$$

$$
\begin{aligned}
b \in \mathscr{M}, & (S+A) \cap[R(1-e)+b] & =S(1-e)+b ; \\
b \in \mathscr{N}, & (S+A) \cap[F(b)+b] & =F(b) \cap S+b ; \\
p \in Y_{2}, & (S+A) \cap[p+A] & =p \cap S+A .
\end{aligned}
$$

Next, the quotients $A_{2} / b_{2}$ are computed. For $b \in \mathscr{N}, F(b) \cap S+b$ is a splitting box ideal. Conditions 3.2(a)-(d) are easily verified with the exception of 3.2(b) that $(S+b) \cap(F(b) \cap S+A)=F(b) \cap S+b$. Let $s+c=r+a \in(S+b) \cap(F(b) \cap S+A)$ with $s \in S, c \in b, r \in F(b) \cap S$, and $a \in A$. Then $s-r=a-c \in S \cap A=Z$. But if $b \in \mathscr{N}$, then $Z \subseteq b$. Thus $a \in b, r+a \in F(b) \cap S+b$ and 3.2(b) holds.

Thus by 3.3 we get

$$
\begin{aligned}
& \frac{S+A}{F(b) \cap S+b} \cong \frac{S}{F(b) \cap S} \times \frac{A}{b}=C \times \frac{A}{b}, \quad S \notin F(b) ; \\
& =\frac{A}{b}, \quad S \subseteq F(b) .
\end{aligned}
$$

For $b \in \mathscr{M}$, we have

$$
\frac{S+A}{S(1-e)+b} \cong \frac{A}{A \cap[S(1-e)+b]}=\frac{A}{b} .
$$

Finally, for $p \in Y_{2}$, since $S \cap A=Z$, since $Z \subseteq p$, and since $S \cap(p \cap S+A)$ $=p \cap S+S \cap A=p \cap S+Z=p \cap S$, it follows that

$$
\frac{S+A}{p \cap S+A} \cong \frac{S}{S \cap(p \cap S+A)}=\frac{S}{p \cap S}
$$

Since $S \triangleleft R$, Prim $S \subseteq\{p \cap S \mid p \in Y\}$, and for any $p \in Y$, either $S \subseteq p$ or $S / p \cap S \cong C$. Since $B_{2} \subset B_{3}$ and $p+A \in B_{3}$ if and only if $Z \subseteq p$, it follows that any $p \in Y$ satisfies:

$$
p+A \in \operatorname{Prim} A_{2} \Leftrightarrow Z \subseteq p \quad \text { and } S \nsubseteq p \Leftrightarrow Z \subseteq p, \quad \frac{S}{p \cap S} \cong C .
$$

If $Z \nsubseteq p$, then $Z \nsubseteq p \cap S, Z \subseteq S$. Thus $S / p \cap S \cong C, S=p \cap S+Z$, and

i.e. $p+A \notin B_{2}$.

$$
A_{2} /(p \cap S+A)=0
$$

4.8. For $I \in P$ define a map $\theta: P \rightarrow B_{3}$ as follows:

$$
\begin{aligned}
\theta(I) & =I \cap(R+A)=A+I \cap R & & \text { if } I \supseteq A, \\
& =\langle I \cap A, F(I \cap A)\rangle & & \text { if } I \nsupseteq A .
\end{aligned}
$$

For any $p \in Y$, either $p+A=R+A$ or $p+A \in B_{3}$. This follows from

$$
\begin{aligned}
\frac{R+A}{p+A} \cong \frac{R}{R \cap(p+A)}=\frac{R}{p+Z} & =\frac{R}{p} \cong C, \quad Z \subseteq p \\
& =\{0\}, \quad Z \nsubseteq p .
\end{aligned}
$$


If $I \in P$, then $p=I \cap R \in Y$. If $I \supseteq A$, then $R+A=I \cap R+A$ cannot hold, for otherwise $1 \in R+A \subseteq I$. Thus $\theta$ is well defined, i.e. either $I \supseteq A$ and $A+I \cap R \in B_{3}$, or $I \notin A$, and $I \cap A \in B$. Note that in the last case $I=(I \cap A)^{\sim} \in \widetilde{B}=\{\tilde{b} \mid b \in B\}$. Thus there is a commutative diagram

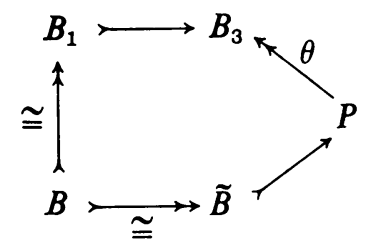

4.9. Lemma. For any $I \in P, \theta(I)=I \cap(R+A)$ and $\theta(I) \cap R=I \cap R$. Furthermore, for any $b \in B, \tilde{b} \cap(R+A)=\langle b, F(b)\rangle$.

Proof. If $A \subseteq I$, then $\theta(I)=I \cap(R+A)$. If $A \nsubseteq I$, then $I=\tilde{b} \in \widetilde{B}$, where $b=I \cap A$. By 2.5(iii), $\tilde{b} A \cup A \tilde{b} \subseteq b$. Clearly, $\langle b, F(b)\rangle \subseteq \tilde{b} \cap(R+A)$. Conversely, let $r-a \in \tilde{b}$ $\cap(R+A)$. If $\langle b, F(b)\rangle=R(1-e)+b$, then

$$
r-a=(r-a)(1-e)+(r-a) e \in r-r e+b+\tilde{b} A .
$$

If $\langle b, F(b)\rangle=b+F(b)$, then for any $x \in A,(r-a) x \in b, x(r-a) \in b$, and $x r=r x$ imply that $a x+b=x a+b$. Thus $a+b \in$ center $A / b=\{0\}$. Hence $a \in b, r \in F(b)$, and $\tilde{b} \cap(R+A)=\langle b, F(b)\rangle$. Thus $\theta(I)=I \cap(R+A)$, and $\theta(I) \cap R=I \cap(R+A) \cap R$ $=I \cap R$.

4.10. Lemma. The map $\theta: P \rightarrow B_{3}$ has the following properties:

(i) $\theta \mid \widetilde{B}: \widetilde{B} \rightarrow$ graph $F$ is a homeomorphism.

(ii) $\theta$ is continuous and open.

Proof. (i) Since $B \rightarrow$ graph $F$ and $B \rightarrow \widetilde{B}$ are homeomorphisms and since there is a commuting diagram of bijective maps

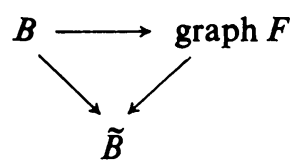

it follows that also $\langle b, F(b)\rangle \rightarrow \tilde{b}$ : graph $F \rightarrow \widetilde{B}$ is a homeomorphism and that the corestriction of $\theta \mid \widetilde{B}$ is simply the inverse of this homeomorphism.

(ii) In view of (i), it suffices to let $\theta(J)=J \cap(R+A)$ with $J \in P$;

$$
N=\left\{b_{3} \in B_{3} \mid \alpha \notin b_{3}\right\}
$$

is a typical neighborhood of $\theta(J)$ where $\alpha \in R+A$ but $\alpha \notin \theta(J)$. Then $I \in P$ satisfies

$$
\theta(I) \in N \Leftrightarrow \alpha \notin \theta(I)=I \cap(R+A) \Leftrightarrow \alpha \in I .
$$


Thus $\theta^{-1}(N)=\{I \in P \mid \alpha \notin I\}$ and $\theta(\{I \in P \mid \alpha \notin I\})=N$. Already $\{I \in P \mid r \notin P\}$ for all $r \in R$ is a basis of open sets for $P$. Thus $\theta$ is continuous and open.

The next result has already been observed in [2] for $B$ and $P$ and in [5] and [4] for $B$ and $B_{3}$.

4.11. The primitive ideal spaces of the algebras $A \subset A_{2} \subset R+A \subset M(A)$ all have isomorphic Stone-Cech compactifications $\beta B \cong \beta B_{2} \cong \beta B_{3} \cong \beta P$.

Proof. For any $C^{*}$-algebra $A$ whatever $(1 \in A$ or $1 \notin A)$ with $\varphi: B \rightarrow M$ the complete regularization of $\operatorname{Prim} A$ and with $R$ the centroid of $A$,

$$
\begin{aligned}
R & \cong C^{b}(B) \cong C^{b}(M), \\
r & \rightarrow \frac{r+b}{b} \rightarrow \frac{r+m}{m}, \quad r \in R, b \in B, m \in M .
\end{aligned}
$$

The assertion follows, since by 1.14, the algebras $A \subset A_{2} \subset A_{3} \subset M(A)$ all have the same centroid $R$. (Note that since $\beta B \cong \beta M$, also $\beta M \cong \beta M_{2} \cong \beta M_{3} \cong \beta M_{4}$.)

4.12. Since $1 \in A_{3}=R+A \subset M(A)$ and since $R=$ center $A_{3}=$ center $M(A)$, it follows that $Y=F_{3}\left(B_{3}\right)=F_{4}(P)$, and that there are bijective maps $Y \rightarrow M_{i}$, $p \rightarrow m_{i}(p), i=3,4$ (see 4.2(ii)). Define a map $j: M_{3} \rightarrow M_{4}$ by $j\left(m_{3}(p)\right)=m_{4}(p)$.

4.13. Lemma. The above map $j: M_{3} \rightarrow M_{4}$ has the following properties.

(i) $j\left(m_{3}\right)=\bigcap\left\{I \mid I \in P, I \cap R=m_{3} \cap R\right\}, m_{3} \in M_{3}$.

(ii) $j: M_{3} \rightarrow M_{4}$ is a homeomorphism.

(iii) There is a commutative diagram:

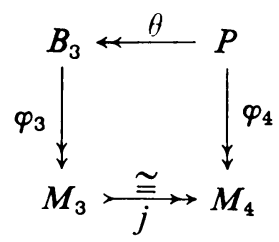

Proof. (i) For any $m_{3} \in M_{3}$, if $m_{3}=m_{3}(p)$ with $p \in Y$, then $m_{3} \cap R=p$ by 4.2(iii). But the definition of $m_{4}(p)$ is $m_{4}(p)=\bigcap\{I \mid I \in P, I \cap R=p\}$ and (i) follows.

(iii) First note that if $A$ is any $C^{*}$-algebra whatever with centroid $R$, and if $\varphi:$ Prim $A \rightarrow M$ is the complete regularization, then for any $b \in \operatorname{Prim} A, \varphi(b) \cap R$ $=b \cap R$. It has to be shown that $j \varphi_{3} \theta=\varphi_{4}$. Since $\theta(J) \cap R=J \cap R$ for $J \in P$, we have

$$
\begin{aligned}
m_{3} & \equiv \varphi_{3} \theta(J)=\bigcap\left\{b_{3} \in B_{3} \mid b_{3} \cap R=\theta(J) \cap R=J \cap R\right\}, \\
j\left(m_{3}\right) & =\bigcap\left\{I \in P \mid I \cap R=m_{3} \cap R\right\} .
\end{aligned}
$$

But $\varphi_{3}\left(b_{3}\right)=m_{3}$ implies $b_{3} \cap R=m_{3} \cap R=J \cap R$, where $b_{3}=\theta(J)$; hence

$$
j \varphi_{3} \theta(J)=\bigcap\{I \in P \mid I \cap R=J \cap R\}=\varphi_{4}(J) .
$$


(ii) To show that $j$ is monic, take two typical points $m_{3}(p)=\varphi_{3}\left(b_{3}\right), m_{3}\left(p^{\prime}\right)$ $=\varphi_{3}\left(b_{3}^{\prime}\right) \in M_{3}$ with $b_{3}, b_{3}^{\prime} \in B_{3}$ and $p, p^{\prime} \in Y$; and suppose $j \varphi_{3}\left(b_{3}\right)=j \varphi_{3}\left(b_{3}^{\prime}\right)$. In the notation of $4.5, m_{3}(p)=m_{3}\left(p^{\prime}\right)$ if and only if $p=p^{\prime}$. Also, $m_{3}(p) \cap R=p$. Thus $\varphi\left(b_{3}\right) \cap R=\varphi\left(b_{3}^{\prime}\right) \cap R$ and $\varphi\left(b_{3}\right) \cap R=b_{3} \cap R=\varphi\left(b_{3}^{\prime}\right) \cap R=b_{3}^{\prime} \cap R$. Consequently $\varphi\left(b_{3}\right)=\varphi\left(b_{3}^{\prime}\right)$ and $j$ is one to one. Now $R=$ center $(R+A)=$ center $M(A)$ and $R \cong C\left(M_{3}\right) \cong C\left(M_{4}\right)$, the ring of continuous complex functions on $M_{3}$ and $M_{4}$. If $r \in R$ and $m_{3} \in M_{3}$, then

$$
r \notin m_{3} \Leftrightarrow r \notin m_{3} \cap R, \quad r \notin j\left(m_{3}\right) \Leftrightarrow r \notin j\left(m_{3}\right) \cap R .
$$

But by definition of $j, j\left(m_{3}\right) \cap R=m_{3} \cap R$. Thus $r \notin m_{3}$ if and only if $r \notin j\left(m_{3}\right)$ and the $\operatorname{map} j$ is a homeomorphism.

The main results of this section, i.e. the description of Prim $M(A)$ and its complete regularization in terms of the known spaces Prim $A$ and Prim $(R+A)$, are recapitulated in the next theorem.

4.14. TheOREM II. Let $A$ be any $C^{*}$-algebra and let the notation be as in 4.1-4.12.

(i) There is a commutative diagram of continuous maps

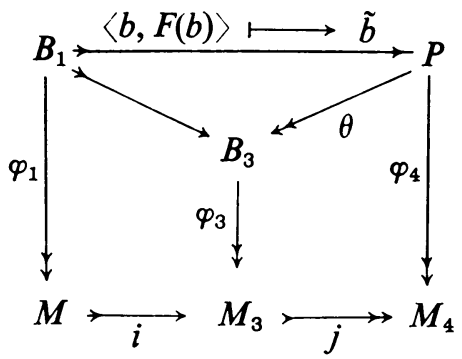

Aside from the usual maps, the maps $\theta, i$, and $j$ are given by

$$
\begin{aligned}
\theta(I) & =I \cap(R+A) & & I \in P, \\
i(m) & =\bigcap\left\{b_{3} \in B_{3} \mid b_{3} \cap R=m \cap R\right\} & & m \in M, \\
j\left(m_{3}\right) & =\bigcap\left\{I \in P \mid I \cap R=m_{3} \cap R\right\} & & m_{3} \in M_{3} .
\end{aligned}
$$

Furthermore, each $b \in B$ satisfies $\tilde{b} \cap(R+A)=\langle b, F(b)\rangle$.

(ii) Viewing $B_{1}$ as a subset $B_{1} \subset$ graph $F \cup\{A\} \times Y$, let $\pi_{1}, \pi_{2}:(B \cup\{A\}) \times Y \rightarrow$ $B \cup\{A\}, Y$ be the projections. Then

$$
B \longrightarrow \widetilde{B} \stackrel{\theta}{\longrightarrow} \operatorname{graph} F \stackrel{\pi_{1}}{\longrightarrow} B
$$

is the identity. Furthermore, for $I, J \in P$

$$
I \cap R \neq J \cap R \Rightarrow \theta(I) \neq \theta(J) .
$$

Thus $\theta$ induces a fibering of $P$ above $Y$. Since $p+A \rightarrow p: B_{3}\left|B_{1} \rightarrow \pi_{2}\left(B_{3}\right)\right| \pi_{2}\left(B_{1}\right) \subset Y$ is bijective, $P$ can be viewed as fibers above $B_{3}$ with the projection $\theta: P \rightarrow B_{3}$ one to one above $B_{1} \subset B_{3}$. 
(iii) $i$ embeds $M$ as a subspace of $M_{3} ; j$ is an isomorphism; the restriction and corestriction $\theta \mid \widetilde{B}: \widetilde{B} \rightarrow B_{1}$ is a homeomorphism.

The next observation completely determines $\pi_{2}\left(B_{3}\right)$ as well as answers a question which was left open in [4]. The proof depends on some technical facts about $C^{*}$-algebras.

4.15. In the above notation, $Y \backslash F(B) \subseteq \pi_{2}\left(B_{3}\right)$; in particular $Z \subseteq p$ for all $p \notin F(B)$.

Proof. For $p_{0} \in Y, p_{0} \notin \pi_{2}\left(B_{3}\right) \Leftrightarrow R=p_{0}+Z$. If so, then $1=z+c$ with $z \in Z$ and $c \in p_{0}$. Let $p_{0} \notin F(B)$. Since for any $b \in B, Z \cap b=Z \cap F(b)$, we have

$$
\frac{Z+b}{b} \cong \frac{Z+F(b)}{F(b)} \subseteq \frac{R}{F(b)}
$$

Since $C^{b}(Y)=R \supset Z,\|z\|=\sup \{\|z+p\| \mid p \in Y, z+p \in R / p \cong C\}$. Also

$$
\|z\|=\sup \{\|z+b\| \mid b \in B\}
$$

holds in any $C^{*}$-algebra. For any real $\lambda>0$ and any element $z$ in a $C^{*}$-algebra $A$, the set $K=\{b \in B \mid\|z+b\| \geqq \lambda\}$ is compact. Consequently, since $F$ is continuous, so is also $F(K) \subset Y$. There is an $r \in R,\|r\| \leqq 1$, such that $r+p_{0}=1+p_{0}$ and $r \in \bigcap F(K)$. Then $1+p_{0}=r z+p_{0}$, while $r z \in r Z \subseteq Z$ with $\|r z\| \leqq \lambda<1$, a contradiction.

4.16. Corollary to Theorem II. If $B=\mathscr{M} \cup \mathscr{N}$ (see 4.7), then

(i) $B_{3}=$ graph $F \cup\{\langle A, p\rangle \mid p \in Y, Z \subseteq p\}$; furthermore

$$
\{p \in Y \mid Z \subseteq p\}=F(\mathscr{N}) \cup(Y \backslash F(B)) \cup\{p \in F(\mathscr{M}) \mid Z \subseteq p\},
$$

(ii) $\theta^{-1}(\langle b, F(b)\rangle)=\{\tilde{b}\}$ for $b \in B$,

(iii) $\theta^{-1}\left(B_{3} \mid B_{1}\right)=\{I \in P \mid A \subseteq I\} \cong \operatorname{Prim} M(A) / A$.

If $\psi: \operatorname{Prim} M(A) / A \rightarrow Y$ is the function $\psi(I / A)=I \cap R$, then

(iv) $\psi(I / A)=F \circ \pi_{1} \circ \theta(I)$; in particular, $\psi$ is continuous.

4.17. Remarks. 1. In 4.16(i) for $p=F(b) \in F(\mathscr{M})$, not only $Z \nsubseteq F(b)$, but also the unexpected possibility $Z \subseteq b$ and $Z \subseteq F(b)$ actually may happen (see Example 4.22).

2. For $p \in \pi_{2}\left(B_{3} \mid B_{1}\right), \psi^{-1}(p)$ need not have a largest element nor does it have to be linearly ordered (see Example 4.21).

In [2], Busby shows that if $A$ is a $C^{*}$-algebra (with or without an identity) and $Z=$ center $A$, that then $\operatorname{Prim} Z$ can be embedded as an open subset of Prim $A$, provided Prim $A$ is Hausdorff. In order to have an embedding, the latter assumption is necessary. The next proposition deals with the case when Prim $A$ is not necessarily Hausdorff. Then afterwards, Busby's result is obtained as a corollary. It seems interesting that even though both the question and the answer could be formulated entirely in terms of $A$ alone without reference to the centroid, the solution requires the use of $R$.

The usual logical symbols " $\exists$ " (there exists) and " $\forall$ " (for any) are used whenever convenient. 
4.18. Lemma. Define $Z(B), Z(M)$, and $Z(Y)$ as the set of ideals of $B, M$, and $Y$ which do not contain $Z$.

(i) For $p \in Y$, conditions (a)-(d) are equivalent:
(a) $\exists b \in B$ such that $F(b)=p$ and $Z \nsubseteq b$.
(b) $\forall b \in B$ such that $F(b)=p, Z \nsubseteq b$.
(c) $Z \nsubseteq m(p)$.
(d) $Z \nsubseteq p$.

(ii) For $p \in Y$ and $m=m(p) \in M$, if (i) holds, then there is an $e \in Z$ that is a relative identity simultaneously for the following ideals:

(e) $\forall b \in B$ such that $F(b)=p, 1=e+b \in A / b$;

(f) $1=e+m \in A / m$;

(g) $1=e+p \in A / p$.

(iii) $\varphi^{-1}(Z(M))=Z(B)$ and $F^{-1}(Z(Y))=Z(B)$.

(iv) $Z(B), Z(M)$, and $Z(Y)$ are open; $Z(Y) \subseteq F(B)$.

Proof. (i) If $b, q \in B$ with $F(b)=F(q)=p$ and $m=m(p) \in M$, then it follows from $Z \cap b=Z \cap q=Z \cap m=Z \cap p$ that $Z|b=Z| q=Z|m=Z| p$. Consequently, (i) follows.

(ii) If (i) holds, choose $q \in B$ with $F(q)=p$ and $\varphi(q)=m=m(p)$. Take $e \in Z \mid q$. Since $q \neq e+q \in$ center $A / q \cong C$, by multiplying $e$ by an appropriate scalar, it may be assumed that $1=e+q \in A / q$. Since $e \in Z \subset R$, and since $(1-e) A \subseteq q$, it follows that $1-e \in F(q)=p=\{r \in R \mid r A \subseteq m\}$. Thus $1=e+p \in R / p$ and $1=e+m \in A / m$. Conclusion (iii) follows easily from (i).

(iv) Clearly, $Z(B)$ is open since $\{b \in B \mid Z \subseteq b\}$ is closed, while $Z(Y)$ is open because $R \cong C^{b}(Y)$. Recall that $R \cong C^{b}(M)$ under $r \rightarrow \tilde{r}$, where at $m=m(p) \in M$,

$$
\tilde{r}(m)=r+m \in \frac{R+m}{m} \cong \frac{R}{p} \cong C .
$$

Thus for any $z \in Z,\{m \in M \mid z \notin m\}=\{m \in M \mid \tilde{z}(m) \neq 0\}$ is open, and hence also $Z(M)$. By 4.15, $Z(Y) \subseteq F(B)$.

4.19. Proposition. Let $A$ be a $C^{*}$-algebra, $B=\operatorname{Prim} A, Z=\operatorname{center} A, \varphi: B \rightarrow M$ the complete regularization of $B$, and $Z(B)=\{b \in B \mid Z \ddagger b\}, Z(M)=\{m \in M \mid Z \ddagger m\}$. Then

(i) $Z(B), Z(M)$ are open, consist of modular ideals and $Z(B)=\varphi^{-1}(Z(M))$.

(ii) $\operatorname{Prim} Z \cong Z(M)$.

Proof. Conclusion (i) follows from the previous lemma. (ii) Then $Z=R \cap A \triangleleft R$, and $\operatorname{Prim} Z \cong Z(Y)=\{p \in Y \mid Z \ddagger p\} \subset Y$ is open. Define $X \subset B_{1}$ by

$$
X=\pi_{2}^{-1}(Z(Y)) \cap B_{1} \text {. }
$$

If $b \in B$ and $p=F(b)$, then $p \in Z(Y)$ if and only if $Z \nsubseteq b$. Since $F(b)=\pi_{2}(\langle b, F(b)\rangle)$, $\pi_{1}(X)=Z(B)$. Since $\pi_{1} \mid$ graph $F$ is a homeomorphism, $Z(B) \cong X$. (Note that for $p \in Z(Y), p+A \notin B_{3}$, and $\pi_{2}(X)=Z(Y)$.) Since $\pi_{2} \mid B_{1}: B_{1} \rightarrow F(B)$ is known to be 
one of the equivalent forms of the complete regularization of $B([4,3.16(4), \mathrm{p}$. 194]), it follows from $Z(B) \cong X$, that $\varphi(Z(B)) \cong \pi_{2}(X)=Z(Y)$.

4.20. Corollary. Suppose that in addition to the hypotheses of the previous proposition, Prim $A$ is Hausdorff. Then $\operatorname{Prim} Z \cong Z(B) \subseteq \operatorname{Prim} A$.

Proof. Since Prim $A$ is always locally compact it is completely regular if and only if it is Hausdorff. In this case $\pi_{2} \mid B_{1}: B_{1} \rightarrow F(B)$ is a homeomorphism and hence $Z(Y) \cong Z(B)$.

A twofold counterexample is given. It shows that $\psi^{-1}(p)$ need not be linearly ordered by inclusion. For $m \in M$, recall that $\tilde{m} \triangleleft M(A)$ is

$$
\tilde{m} \equiv\{T \in M(A) \mid T A \cup A T \subseteq m\}=\bigcap\{\tilde{b} \mid \varphi(b)=m\} \supseteq j(i(m)) .
$$

(Note that if $m=m(p)$, then $\tilde{m}=\{T \in M(A) \mid T A \subseteq b$, all $b \in B$ with $F(b)=p\}$ $=\bigcap\{\tilde{b} \mid b \in B, F(b)=p\}$.) The example also shows that the conjecture $j(i(m))=\tilde{m}$ is false.

4.21. Example. Consider a Hilbert space $H=H_{1} \oplus H_{2} \oplus H_{3}$ which is an orthogonal sum of three mutually perpendicular closed infinite dimensional subspaces $H_{1}, H_{2}$, and $H_{3}$. The bounded and the compact operators are denoted by $L H$ and $L C H ; L H_{i}$ and $L C H_{i} \subset L C H$ denote operators leaving $H_{i}$ invariant and which are zero on $H_{i}^{\perp}$. Consider the ring $A$ of all continuous functions $g:[0,1] \rightarrow$ $L C H$ such that $g(1)=g_{1}+g_{2} \in L C H$, where $g_{i} \in L C H_{i}$. Then $M(A)$ consists of all continuous functions $G:[0,1] \rightarrow L H$ such that $G(1)=G_{1}+G_{2}+G_{3}$, where $G_{i} \in L H_{i}$. Small letters are used for $A$, capitals for $M(A)$. Note that $g_{i} G_{j}=G_{j} g_{i}=0$ for $i \neq j$. The centroid $R$ consists of all $\alpha 1$, where $1: H \rightarrow H$ is the identity and $\alpha:[0,1] \rightarrow C$ is continuous. Thus $Y=\{p(t) \mid 0 \leqq t \leqq 1\}$ where $p(t)=\{\alpha \mathbf{1} \mid \alpha(t)=0\}$. For $0 \leqq t<1$, define $b(t), I_{0}(t)$, and $I_{1}(t)$ by

$$
\begin{aligned}
b(t) & =\{g \in A \mid g(t)=0\} ; \\
I_{0}(t) & =\{G \in M(A) \mid G(t)=0\} \subset I_{1}(t)=\{G \in M(A) \mid G(t) \in L C H\} .
\end{aligned}
$$

While at $t=1$, define $b^{k}, I_{0}^{k}$, and $I_{1}^{k}$ to be

$$
\begin{aligned}
& b^{k}=\left\{g \in A \mid g_{k}=0\right\}, \quad k=1,2 ; \\
& I_{0}^{k}=\left\{G \in M(A) \mid G_{k}=0\right\} \subset I_{1}^{k}=\left\{G \in M(A) \mid G_{k} \in L C H_{k}\right\}, \quad k=1,2,3 .
\end{aligned}
$$

Thus $B=\{b(t) \mid 0 \leqq t<1\} \cup\left\{b^{1}, b^{2}\right\}$ and

$$
P=\left\{I_{0}(t), I_{1}(t) \mid 0 \leqq t<1\right\} \cup\left\{I_{j}^{k} \mid j=0,1 ; k=1,2,3\right\} .
$$

Since a typical hull-kernel neighborhood of $b^{k}$ is of the form $\{b(t) \mid c<t<1\}$ $\cup\left\{b^{k}\right\}$ for some $0<c<1$, it follows that any two hull-kernel neighborhoods of $b^{1}, b^{2}$ intersect; $\psi^{-1}(p(1))=\left\{I_{j}^{k} \mid j=0,1 ; k=1,2,3\right\}$, but any three ideals with one out of each of the pairs

$$
I_{0}^{1} \subset I_{1}^{1} ; \quad I_{0}^{2} \subset I_{1}^{2} ; \quad I_{0}^{3} \subset I_{1}^{3}
$$


are not ordered by inclusion. Also any two neighborhoods (they are of the same form as those of $b^{k}$ ) of $I_{j}^{k}$ and $I_{p}^{r}$ for any $k, j, r, p$ intersect. Now

$$
M=\{b(t) \mid 0 \leqq t<1\} \cup\left\{b^{1} \cap b^{2}\right\},
$$

while $M_{4}=\left\{I_{0}(t) \mid 0 \leqq t<1\right\} \cup\left\{I_{0}^{1} \cap I_{0}^{2} \cap I_{0}^{3}\right\}$. For $0 \leqq t<1, \quad \tilde{b}(t)=I_{0}(t)$, while $\left(b^{1} \cap b^{2}\right)^{\sim}=\left\{G \mid G A \cup A G \subseteq b^{1} \cap b^{2}\right\}=\left\{G \mid G_{1}=G_{2}=0\right\} \notin M_{4}$ since $j\left(b^{1} \cap b^{2}\right)=$ $I_{0}^{1} \cap I_{0}^{2} \cap I_{0}^{3}=\left\{G \mid G_{1}=G_{2}=G_{3}=0\right\}$. This example is noteworthy in that

$$
\text { center }(M(A) / A) \neq(A+R) / A \text {. }
$$

Here $(R+A) / A \cong C$, the complex numbers, while

$$
M(A) / A \cong\left(L H_{1} / L C H_{1}\right) \oplus\left(L H_{2} / L C H_{2}\right) \oplus L H_{3} .
$$

Thus center $(M(A) / A) \cong C \oplus C \oplus C$.

The next example shows that it is actually possible that $Z \subseteq m \in M=B$, but that $m$ nevertheless is still modular (see 4.17,1).

4.22. ExAmple. Again let $L C H$ denote the compact operators on some infinite dimensional Hilbert space; let $H_{1} \subset H$ be a finite dimensional subspace. Let $A$ be the ring of all continuous functions $f:[0,1] \rightarrow L C H$ such that $f(1) \mid\left(H_{1}^{\perp}\right)=0$ and $f(1) H_{1} \subseteq H_{1}$. For $0 \leqq x \leqq 1$, let $b(x)=\{f \in A \mid f(x)=0\}$. Then

$$
M=B=\{b(x) \mid 0 \leqq x \leqq 1\} .
$$

center $A=\{0\} \subseteq b(1)$, while $b(1)$ is modular.

5. Classification of abelian extensions. Only extensions of the form $S+A$ where $S$ is an ideal of the centroid will be considered here. The more general kinds of extensions where $S$ need not act faithfully on $A$ will not be considered. The notation, definitions, and conclusions of $\S 4$ will be used as well as [4].

5.1. When talking simultaneously about $p$ and $m_{i}$, it will be tacitly assumed and it will be abundantly clear from the context that $p$ and $m_{i}$ are related by

$$
m_{i}=m_{i}(p)=\bigcap\left\{b_{i} \cap A_{i} \mid b_{i} \in B_{i} ; F_{i}\left(b_{i}\right)=p\right\}, \quad i=1,2,3 ;
$$

the subscript one is sometimes omitted in $A=A_{1}, M=M_{1}$, and $m=m_{1}$. This definition uses the fact 1.14 that the centroid of $A_{2}$ and $A_{3}$ is also $R$. Note that for $i=3$ the above becomes

$$
m_{3}=\bigcap\left\{b_{3} \mid b_{3} \in B_{3} ; b_{3} \cap R=p\right\} .
$$

It may be helpful to observe that it will be necessary to complete the diagram in 4.5 with injective maps also along the bottom rows; it is no longer possible to obtain $M_{1}$ and $M_{2}$ simply by intersecting certain distinguished elements of $M_{3}$ with $A_{1}$ and $A_{2}$ as is the case with the $B_{i}$. The next definition among other things will accomplish this.

5.2. Definition. For any $p \in F(B)$, define an ideal $m^{\prime}$ of $A_{3}$ by

$$
m^{\prime}=m^{\prime}(p)=\bigcap\{\langle b, F(b)\rangle \mid b \in B, F(b)=p\} .
$$


Again it will be clear from the context that when $m^{\prime}$ and $p$ are used simultaneously that they are related as above. Define $M^{\prime}=\left\{m^{\prime}(p) \mid p \in Y\right\}$.

The next lemma is easier to visualize if $B_{2}$ is viewed as a subset $B_{2} \subset B^{\prime} \times Y$.

5.3. Lemma. Each $b_{2} \in B_{2}$ with $A \nsubseteq b_{2}$ satisfies $F_{2}\left(b_{2}\right)=F\left(b_{2} \cap A\right)=F\left(\pi_{1}\left(b_{2}\right)\right)$.

Proof. By 4.4,

$$
F_{2}\left(b_{2}\right) \equiv\left\{r \in R \mid r A_{2} \subseteq b_{2} \cap A_{2}\right\}=\left\{r \in R \mid r A_{2} \subseteq b_{2}\right\}=b_{2} \cap R .
$$

Set $b=b_{2} \cap A \in B$. For $r \in F_{2}\left(b_{2}\right), r A \subseteq A$ since $A \triangleleft R+A$; thus $r A \subseteq b_{2} \cap A=b$. Consequently

$$
F(b) \equiv\{r \in R \mid r A \subseteq b\} \supseteq F\left(b_{2}\right) .
$$

But both $F(b)$ and $F_{2}\left(b_{2}\right)$ are maximal ideals of $R$. Hence

$$
F_{2}\left(b_{2}\right)=F\left(b_{2} \cap A\right)=F\left(\pi_{1}\left(b_{2}\right)\right) \text {. }
$$

In (ii) of the next lemma, in particular $A_{2}$ may be taken as $A_{2}=A_{3}$, in which case $S=R, B_{2}=B_{3}$, and $M_{2}=M_{3}$.

5.4. Lemma. The sets of ideals $M_{1}, M_{2}$, and $M_{3}$ can be constructed from $M^{\prime}$ and $Y$ as follows:

(i) $M_{1}=\left\{m^{\prime} \cap A \mid m^{\prime} \in M^{\prime}\right\}$;

(ii) $M_{2}=\left\{m^{\prime}(p) \cap A_{2} \mid p \in F(B), A+p \notin B_{2}\right\}$

$$
\begin{aligned}
& \cup\left\{m^{\prime}(p) \cap A_{2} \cap(A+p) \mid p \in F(B), A+p \in B_{2}\right\} \\
& \cup\left\{(A+p) \cap A_{2} \mid p \notin F(B), A+p \in B_{2}\right\} .
\end{aligned}
$$

(iii) There is a commutative diagram where all the maps except possibly $\varphi_{1}, \varphi_{2}$, and $\varphi_{3}$ are monic while $j_{1}$ is an isomorphism.

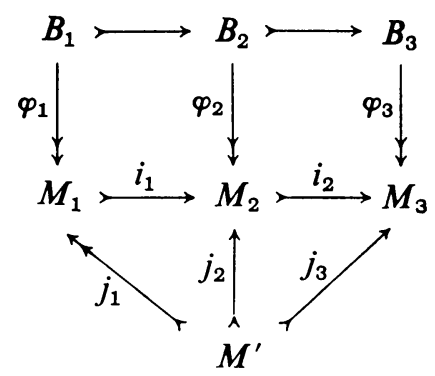

(iv) $\bigcap M^{\prime}=\bigcap i_{1}\left(M_{1}\right)=\bigcap i_{2} i_{1}\left(M_{1}\right)=\bigcap i_{2}\left(M_{2}\right)=\{0\}$.

Proof. (i) Since $\langle b, F(b)\rangle \cap A=b$, (i) follows.

(ii) By definition, $m_{2}(p)=\bigcap\left\{b_{2} \cap A_{2} \mid b_{2} \in B_{2}, F_{2}\left(b_{2}\right)=p\right\}$. By 5.3, if $\langle b, F(b)\rangle$ appears in the intersection defining $m^{\prime}(p)$, then $\langle b, F(b)\rangle \cap A_{2}=b_{2}$ also appears in the intersection defining $m_{2}(p)$. The latter intersection, however, may or may not include $A+p$. Finally, if $p \notin F(B)$, then the intersection defining $m_{2}(p)$ becomes trivial, i.e. $m_{2}(p)=(A+p) \cap A_{2}$. 
(iii) Define $i_{1}$ and $i_{2}$ by $i_{1}\left(m_{1}(p)\right)=m_{2}(p), i_{2}\left(m_{2}(p)\right)=m_{3}(p)$. If $p_{1}, p_{2} \in F(B)$ and $m^{\prime}\left(p_{1}\right)=m^{\prime}\left(p_{2}\right)$, then $m\left(p_{1}\right)=m^{\prime}\left(p_{1}\right) \cap A=m^{\prime}\left(p_{2}\right) \cap A=m\left(p_{2}\right)$. Thus by 4.2(ii), $p_{1}=p_{2}$ and $j_{1}$ is bijective. Again 5.3 shows that the diagram commutes. By 4.2(ii) applied to $A_{i}$, the map $F_{i}\left(B_{i}\right) \rightarrow M_{i}, p \rightarrow m_{i}(p)$ is bijective. Consequently $j_{1}, j_{2}, j_{3}$, $i_{1}$, and $i_{2}$ are monic.

(iv) Since $\cap \widetilde{B}=\{\alpha \in M(A) \mid \alpha A \subseteq \bigcap B=0\}=\{0\}$, and since $B_{1}=\left\{\tilde{b} \cap A_{3} \mid \tilde{b} \in \tilde{B}\right\}$, also $\bigcap B_{1} \subseteq \bigcap \widetilde{B}=\{0\}$ (i.e. $B_{1}$ is hull-kernel dense in $B_{3}$ ). However, $\bigcap M^{\prime}=\bigcap B_{1}$, and so $\bigcap M^{\prime}=\{0\}$. Since

$$
\bigcap i_{1}\left(M_{1}\right), \bigcap i_{2} i_{1}\left(M_{2}\right), \bigcap i_{2}\left(M_{2}\right) \subseteq \bigcap M^{\prime}
$$

it follows that all these intersections are zero.

5.5. If $p \in F(B) \subset F_{2}\left(B_{2}\right)$ and $p_{2} \in F_{2}\left(B_{2}\right) \subset F_{3}\left(B_{3}\right)=Y$, then

(i) $m_{1}(p)=m_{2}(p) \cap A_{1}$;

(ii) $m_{2}\left(p_{2}\right)=m_{3}\left(p_{2}\right) \cap A_{2}$;

(iii) in particular, $i_{2}\left(m\left(p_{2}\right)\right) \cap A_{2}=m\left(p_{2}\right)$ and $i_{1}(m(p)) \cap A=m(p)$.

Proof. (i) Since $(A+p) \cap A_{2} \cap A=A$, in either of the two possibilities for $m_{2}(p)$ in 5.4(ii), we get $m_{2}(p) \cap A=m^{\prime}(p) \cap A=m(p)$.

(ii) and (iii). Use of 5.4(ii) with $A_{2}=A_{3}$ gives $m_{3}$. If $p_{2} \notin F(B)$, then $m_{3}\left(p_{2}\right)$ $=p_{2}+A$ and $m_{2}\left(p_{2}\right)=\left(p_{2}+A\right) \cap A_{2}=m_{3}\left(p_{2}\right) \cap A_{2}$. Let $p_{2}=p \in F(B)$ and consider the following cases.

Case 1. $m_{3}(p)=m^{\prime}(p)$ with $A+p \notin B_{3}$. Then $A+p \notin B_{2}$, and

$$
m_{2}(p)=m^{\prime}(p) \cap A_{2}=m_{3}(p) \cap A_{2} .
$$

Case 2. $m_{3}(p)=m^{\prime}(p) \cap(A+p)$ with $A+p \in B_{3} \mid B_{2}$. Then $m_{2}(p)=m^{\prime}(p) \cap A_{2}$. But since $A_{2} \subseteq A+p$, we have $A_{2} \cap(A+p)=A_{2}$ and again $m_{2}(p)=m_{3}(p) \cap A_{2}$.

Case 3. $m_{3}(p)=m^{\prime}(p) \cap(A+p), A+p \in B_{2}$. Thus

$$
m_{2}(p)=m^{\prime}(p) \cap A_{2} \cap(A+p)=m_{3}(p) \cap A_{2} .
$$

Each $A_{2} / b_{2}$ was concretely identified in 4.7 ; in order to identify $A_{2} / m_{2}$, first $m^{\prime}$ and then $m_{2}=j_{2}\left(m^{\prime}\right)$ have to be described (see [4, 1.5(5)-(8), p. 179] and [4, 3.18(1), p. 196]).

5.6. LEMMA. The ideal $m^{\prime}=m^{\prime}(p) \in M^{\prime}$ can be expressed in terms of $m=m(p) \in M$ as follows:

(i) $1 \notin A / m \Leftrightarrow m^{\prime}=p+m$;

(ii) $e \in A, 1=e+m \in A / m \Leftrightarrow m^{\prime}=R(1-e)+m$.

Proof. Since $p+m \subseteq\langle b, F(b)\rangle$ and $\langle b, F(b)\rangle \cap A=b$, it follows that

$$
p+m \subseteq m^{\prime}=\bigcap\{\langle b, F(b)\rangle \mid F(b)=p\} \quad \text { and } \quad m^{\prime} \cap A=m .
$$

Since the projection $\left(m^{\prime}+A\right) \cap R$ of $m^{\prime}$ into $R$ satisfies $p \subseteq\left(m^{\prime}+A\right) \cap R \triangleleft R$, by 
maximality of $p,\left(m^{\prime}+A\right) \cap R=p$ or $R$. Thus by $3.6, m^{\prime}$ is of one of the following two mutually exclusive forms

(i') $\left(m^{\prime}+A\right) \cap R=p \Rightarrow m^{\prime}=p+m$,

(ii') $\left(m^{\prime}+A\right) \cap R=R \Rightarrow m^{\prime}=R(1-e)+m$.

But then (i) and (ii) of the lemma follow from

$$
\begin{aligned}
1=e+m \in A / m & \Leftrightarrow \forall b \in B \text { with } p=F(b),\langle b, F(b)\rangle=R(1-e)+b \\
& \Leftrightarrow m^{\prime}=\bigcap\{R(1-e)+b \mid b \in B, F(b)=p\} \\
& \Leftrightarrow m^{\prime}=R(1-e)+m .
\end{aligned}
$$

To prove the latter, let $b$ and $q \in B$ with $F(b)=F(q)=p$. Let

$$
r(1-e)+a=s(1-e)+c \in[R(1-e)+b] \cap[R(1-e)+q]
$$

where $r, s \in R, a \in b$ and $c \in q$. It suffices to show that $c \in b$. Since $e$ is an identity modulo both $b$ and $q$, it follows that $r(1-e), s(1-e) \in \tilde{b}$, where

$$
\tilde{b} \equiv\{\alpha \in M(A) \mid \alpha A \subseteq b\} .
$$

But then $c=r(1-e)-s(1-e)+a \in \tilde{b} \cap A=b$.

The previous conditions $1 \notin A / m$ or $1 \in A / m$ are useless for applications (see $[4,3.17$, p. 196]).

5.7. LEMMA. With the above notation, the following hold:

(i) $m^{\prime}=m+p \Leftrightarrow m$ is not modular in $A \Rightarrow Z \subseteq m$.

(ii) $m^{\prime}=R(1-e)+m \Leftrightarrow$ (a) either $Z \ddagger m$, (b) or $Z \subseteq m$ but $m$ is nevertheless still modular in $A$.

Proof. In view of 5.6, it merely suffices to show that if $Z \nsubseteq m$, then $m$ is modular. Since $Z \ddagger m=\bigcap\{b \in B \mid F(b)=p\}$, there is a $b \in B$ with $Z \nsubseteq b$ and $F(b)=p$. Since all $b$ appearing in the above intersection satisfy $p=F(b)=\{r \in R \mid r A \subseteq b\}$, it follows that $F(b)=\{r \in R \mid r A \subseteq m\}$. Since $b=L: A$ for some regular maximal left ideal $L$, we have $Z A \nsubseteq b$; hence $Z A \nsubseteq m$, and consequently $Z \nsubseteq p$. Thus $R=p+Z$. If $1=c+e$, $c \in p, e \in Z$, then $c A \subseteq p A \subseteq m$ and $e$ is an identity for $A$ modulo $m$.

The next observation will be used frequently.

5.8. Corollary. No matter whether $m^{\prime}$ is of the form (i) or (ii) in 5.7,

$$
m^{\prime} \cap(p+A)=p+m .
$$

Proof. If $r+a \in[R(1-e)+m] \cap(p+A)$ with $r \in p, a \in A$ then $r e \in r A \subseteq m$ implies $r \in R(1-e)+m$. But then $a \in[R(1-e)+m] \cap A=m$ and $r+a \in p+m$.

At last $m_{2}(p)$ can be computed.

5.9. Proposition. Each $m_{2}(p) \in M_{2}$ with $m=m(p) \in M$ and $m^{\prime}(p) \in M^{\prime}$ satisfies:

(i) For $p+A \notin B_{2}, m_{2}(p)=m^{\prime}(p) \cap A_{2}=p \cap S+m \quad$ if 5.7(i) holds;

$$
=S(1-e)+m \text { if 5.7(ii) holds. }
$$


(ii) For $p+A \in B_{2} ; m_{2}(p)=m^{\prime}(p) \cap A_{2} \cap(p+A)=p \cap S+m$.

(iii) $m_{2}(p)=p \cap S+m \Rightarrow Z \subseteq m$ (converse may fail).

By specializing $A_{2}=R+A$ in the above proposition, a complicated result from $[4,3.21(1)$, p. 197] follows very simply. In the next corollary, as well as in subsequent proofs, the four implications in (a) and (b) below can be reduced to two by observing that $m_{3}$ is necessarily of one of two mutually exclusive forms.

5.10. CoRollaRy. Each $m_{3}=m_{3}(p) \in M_{3}$ and $m=m(p) \in M$ with $p \in Y$ satisfies:

(a) $Z \nsubseteq m \Leftrightarrow m_{3}=R(1-e)+m$,

(b) $Z \subseteq m \Leftrightarrow m_{3}=p+m$.

Proof. First note that

$$
Z \subseteq m \Leftrightarrow Z \subseteq p \Leftrightarrow p+A \in B_{3} .
$$

If 5.9(i) holds then $Z \nsubseteq m$, and by 5.7(ii)(a), $m_{3}=R(1-e)+m$. If 5.9(ii) applies, then $Z \subseteq m, p+A \in B_{3}$ and $m_{3}=p+m$ by 5.8 .

Conclusions (ii) and (iii) in the next lemma are established in slightly greater generality than actually later needed.

5.11. LEMMA. If $m=m(p) \in M, m_{2}=m_{2}(p) \in M_{2}$ with $p \in Y$, then

$$
m_{2}=S(1-e)+m \Rightarrow \frac{A_{2}}{m_{2}} \cong \frac{A}{m} .
$$

Now assume further that $Z \subseteq m$. The latter holds in particular if $m_{2}=p \cap S+m$ (see 5.9(iii)). Then

(ii) $p \cap S+m$ is a splitting box ideal (see 3.2);

(iii) consequently

$$
\frac{S+A}{p \cap S+m} \cong \frac{S}{p \cap S} \times \frac{A}{m}
$$

$$
\frac{S}{p \cap S} \cong C \Leftrightarrow S \nsubseteq p \Leftrightarrow p+A \in B_{2} \Rightarrow m_{2}=p \cap S+m .
$$

Proof. (i) If $m_{2}=S(1-e)+m$, then 5.9 shows that $p+A \notin B_{2}$ and $m_{2}=m^{\prime} \cap A_{2}$. Furthermore $m^{\prime}=R(1-e)+m$, and $A+m^{\prime}=R+A$. Thus

$$
\frac{A_{2}}{m_{2}}=\frac{A_{2}}{m^{\prime} \cap A_{2}} \cong \frac{A_{2}+m^{\prime}}{m^{\prime}}=\frac{R+A}{m^{\prime}}
$$

Since $A \cap m^{\prime}=m$, it follows that

$$
\frac{A+m^{\prime}}{m^{\prime}} \cong \frac{A}{A \cap m^{\prime}}=\frac{A}{m} .
$$

Conclusion (iv) is immediate while (iii) follows from (ii). Condition 3.2(a) holds with $S_{1}=p \cap S, A_{1}=m$. (b) First, $p \cap S+m \subseteq(S+m) \cap(p \cap S+A)$. Conversely, suppose $s+n=r+a$ belongs to this intersection with $s \in S, n \in m, r \in p \cap S$, and $a \in A$. Then $a-n=s-r \in S \cap A=Z \subseteq m$. Thus $a \in m$ and $r+a \in p \cap S+m$. Hence 
(b) holds. Since $(p \cap S) A \subseteq m$, condition (c) $(p \cap S+m) \cap A=m$ follows. Next, if $s=r+c \in S \cap(p \cap S+m)$ with $s \in S, r \in p \cap S$, and $c \in m$, then $c=s-r \in m \cap S$ $=m \cap Z=p \cap Z \subseteq p \cap S$. Thus $s \in p \cap S$ and (d) $S \cap(p \cap S+m)=p \cap S$ holds.

5.12. Remarks. 1. $A_{2} \cong S \times A /\{(z,-z) \mid z \in Z\}, \quad S \cap A=Z$.

2. It may happen in the above proof that $Z \subseteq m$, but nevertheless $m$ is modular with $m_{2}=S(1-e)+m$. In this case $p \cap S+m$ is still a splitting box ideal although $m_{2} \neq p \cap S+m$.

For the readers convenience the foregoing results about the quotients $A_{2} / m_{2}$ are summarized below.

5.13. Proposition. For $A_{2}=S+A, Z=$ center $A, m=m(p) \in M, p \in Y$, the ideal $m_{2}=m_{2}(p) \in M_{2}$ is one of the two ((i) and (ii)) mutually exclusive forms and satisfies the following:

(i) $m_{2}=p \cap S+m \Rightarrow A_{2} / m_{2} \cong S /(p \cap S) \times(A / m) ; Z \subseteq p$ (and $\left.Z \subseteq m\right)$. Furthermore,

$$
\begin{aligned}
& Z \subseteq p, A_{2} \ddagger p+A \Leftrightarrow S /(p \cap S) \cong C ; \\
& Z \subseteq p, A_{2} \subseteq p+A \Leftrightarrow S /(p \cap S)=\{0\} .
\end{aligned}
$$

(ii) $m_{2}=S(1-e)+m \Rightarrow A_{2} / m_{2} \cong A / m$.

Specialization of $A_{2}$ as $A_{2}=R+A$ above together with 5.11 immediately yields a result of [4, 3.21(2), p. 197].

5.14. Corollary. If $m_{3}=m_{3}(p) \in M_{3}$, then

$$
\begin{aligned}
& \frac{A_{3}}{m_{3}} \cong C \times A / m \Leftrightarrow m_{3}=p+m \Leftrightarrow Z \subseteq m . \\
& \frac{A_{3}}{m_{3}} \cong \frac{A}{m} \Leftrightarrow m_{3}=R(1-e)+m \Leftrightarrow Z \ddagger m .
\end{aligned}
$$

The next classification of cases will simplify the solution of the present problem.

\subsection{Consider}

Case 1. $A+p \notin B_{2}$. Case 2. $A+p \in B_{2}$, and subdivide Case 1 further as follows:

Case 1(a). $m^{\prime}=m+p$,

Case 1(b). $m^{\prime}=R(1-e)+m$, where $e+m=1 \in A / m$.

If $A+p \notin B_{2}$, then $m_{2}=m^{\prime} \cap A_{2}$, while if $A+p \in B_{2}$, then $m_{2}=m^{\prime} \cap A_{2} \cap(A+p)$; thus

Case $1(\mathrm{a}) \Rightarrow m_{2}=m+p \cap S$

Case $1(\mathrm{~b}) \Rightarrow m_{2}=S(1-e)+m$,

Case $2 \Rightarrow m_{2}=m+p \cap S$.

Note also that $m^{\prime}=m+p$ implies $m_{3}=m+p$ and $Z \subseteq m$ (see 5.7(i)) and thus $A+p \in B_{3}$ by 4.3 and 4.7. Case 1(b) holds if either $Z \nsubseteq m$ (in which case $A+p \notin B_{3}$ ) or even if $Z \subseteq m$, but $m$ is still modular. Finally, in Case $2, A+p \in B_{2} \subseteq B_{3}$ so that then $Z \subseteq m$.

5.16. Utilizing the previous notation the tables below summarize all of the 
information contained in the previous lemmas concerning $M_{2}, M_{3}$ and the quotients $A_{2} / m_{2}, A_{3} / m_{3}$, as well as the inclusion relations among $Z, m$, and among $S, p$.

\begin{tabular}{|c|c|c|c|c|}
\hline$A+p$ & $Z$ and $m$ & $S$ and $p$ & $A_{2} / m_{2}$ & $A_{3} / m_{3}$ \\
\hline$A+p \notin B_{3}$ & $Z \nsubseteq m$ & $S \subseteq p$ & $A / m$ & $A / m$ \\
\hline$A+p \in B_{3} \mid B_{2}$ & $Z \subseteq m$ & $S \subseteq p$ & $A / m$ & $C \times A / m$ \\
\hline$A+p \in B_{2}$ & $Z \subseteq m$ & $S \nsubseteq p$ & $C \times A / m$ & $C \times A / m$ \\
\hline
\end{tabular}

\begin{tabular}{l|l|l|l|l|l}
$A+p$ & $m$ modular & Case & $m^{\prime}$ & $\frac{m_{2}(p)}{S(1-e)+m}$ & $\frac{m_{3}(p)}{R(1-e)+m}$ \\
\hline$A+p \notin B_{3}$ & yes & $1(\mathrm{~b})$ & $R(1-e)+m$ & $\frac{S(1-e)+m}{m+p}$ \\
\hline$A+p \in B_{3} \mid B_{2}$ & yes & $1(\mathrm{~b})$ & $R(1-e)+m$ & $S(1-e)$ \\
\hdashline$A+p \in B_{3} \mid B_{2}$ & no & $1(\mathrm{a})$ & $m+p$ & $m+p \cap S$ & $m+p$ \\
\hline$A+p \in B_{2}$ & yes & $?$ & $R(1-e)+m$ & $m+p \cap S$ & $m+p$ \\
\hdashline$A+p \in B_{2}$ & no & $?$ & $m+p$ & $m+p \cap S$ & $m+p$ \\
\hline
\end{tabular}

Finally, the solution of the problem is summarized in the next theorem.

5.17. TheOREM III. Consider a $C^{*}$-algebra $A$ without an identity and with centroid $R$. Suppose $A_{2}$ is any closed ideal in $A \subset A_{2} \subset R+A$. Let $M, M_{2}$, and $M_{3}$ be the canonical spaces of ideals obtained by the complete regularization of the respective primitive ideal spaces. For $m \in M$, let $p=\{r \in R \mid r A \subseteq m\}$ and denote $m(p)$ by $m$. Write $M=M(1) \cup M(2) \cup M(3)$ as a disjoint union of subsets

$$
\begin{aligned}
& M(1)=\{m \mid Z \ddagger m\}, \\
& M(2)=\left\{m \mid Z \subseteq m(p), A_{2} \subseteq A+p\right\}, \\
& M(3)=\left\{m \mid Z \subseteq m, A_{2} \ddagger A+p\right\} .
\end{aligned}
$$

(i) There are injective maps

$$
M \stackrel{i_{1}}{\longrightarrow} M_{2} \stackrel{i_{2}}{\longrightarrow} M_{3} .
$$

Furthermore $\bigcap M=\bigcap i_{1}(M)=\bigcap i_{2} i_{1}(M)=\{0\}$.

$$
\begin{aligned}
m \in M(1) & \Rightarrow \frac{A}{m}=\frac{A_{2}}{i_{1}(m)}=\frac{R+A}{i_{2} i_{1}(m)} \\
m \in M(2) & \Rightarrow \frac{A}{m}=\frac{A_{2}}{i_{1}(m)} \neq \frac{R+A}{i_{2} i_{1}(m)}=C \times \frac{A}{m} \\
m \in M(3) & \Rightarrow \frac{A}{m} \neq \frac{A_{2}}{i_{1}(m)}=C \times \frac{A}{m}=\frac{R+A}{i_{2} i_{1}(m)} \\
m_{2} \in M_{2} \mid i_{1}(M) & \Rightarrow \frac{A_{2}}{m_{2}}=C \\
m_{3} \in M_{3} \mid i_{2} i_{1}(M) & \Rightarrow \frac{R+A}{m_{3}}=C .
\end{aligned}
$$


5.18. Remark. The fact that $A_{2}$ is a subdirect product of $\prod\left\{A_{2} / m_{2} \mid m_{2} \in M_{2}\right\}$ does not tell the whole story (see [3]). Each $a \in A_{2}$ defines a function $M_{2} \rightarrow$ reals, $m_{2} \rightarrow\left\|a+m_{2}\right\|$. Suppose $M_{2}$ is endowed with the complete regularization topology. If $1 \notin A_{2}$, i.e. if $A_{2} \neq A_{3}$, then each such function tends to zero outside of compact subsets of $M_{2}$.

5.19. Corollary to TheOREM III. Under the hypotheses and in the notation of the previous theorem, if $M, M_{1}, M_{2}$ are endowed with the complete regularization topology, then

(i) $i_{1}, i_{2}$, and $i_{2} i_{1}$ are embeddings;

(ii) $i_{1}(M)$ is dense in $M_{2}$;

(iii) $i_{2} i_{1}(M)$ is dense in $M_{3}$;

(iv) If $Z=$ center $A$, then $M(1) \cong \operatorname{Prim} Z$.

Proof. Conclusions about $i_{2} i_{1}$ (that is (iii) and part of (i)) follow immediately from 4.6, while the method of proof of 4.6 also works with $M_{3}$ replaced by $M_{2}$. Conclusion (iv) follows from 4.19.

In conclusion, a simple example is given where the conclusions of the theorem as well as those of the corollary may be checked directly.

5.20. EXAmple. Let $X$ be a locally compact Hausdorff space, $H$ an infinite dimensional Hilbert space, and LCH the compact operators on $H$. Let

$$
A=C_{0}(X, \mathrm{LCH})
$$

be the ring of all continuous functions $g: X \rightarrow \mathrm{LCH}$ such that $\|g(x)\|$ tends to zero for $x$ outside of compact sets. Then $R$ is the ring $C^{b}(X)$ of all bounded continuous complex-valued functions on $X$. The typical element of $R+A$ will be written as $g+G 1$ where $g \in A, G \in R$, and $1: H \rightarrow H$ is the identity. Let $g^{\beta}$ and $G^{\beta}$ denote the extensions of $g$ and $G$ to the Stone-Čech compactification $\beta X$ of $X$, where $g^{\beta} \mid(\beta X \backslash X)=0$. For $t \in \beta X$, let $p(t)=\left\{G \in R \mid G^{\beta}(t)=0\right\} \subset R$ and $b(t)=$ $\left\{g \in A \mid g^{\beta}(t)=0\right\} \subseteq A$. Note that for $t \in \beta X \mid X, b(t)=A$. Thus $\operatorname{Prim} A=B=M$ $=\{b(x) \mid x \in X\}$ and Prim $R=Y=\{p(t) \mid t \in \beta X\}$. The function $F: B \rightarrow Y$ is given by $F(b(x))=p(x)$, so that $F(B) \neq Y$. In this case $M=B \cong X$, while

$$
B_{3}=M_{3}=\{b(t)+p(t) \mid t \in \beta X\},
$$

where $b(t)+p(t)$ consists simply of all functions vanishing at $t$. Suppose now that $A_{2}$ is a closed ideal in $A \subset A_{2} \subset R+A$. Then $Z=\{0\}$ and hence $M(1)=\varnothing$. Thus

$$
\begin{aligned}
M(2) & =\left\{b(x) \in M \mid x \in X, \forall g+G \mathbf{1} \in A_{2}, G(x)=0\right\} \\
& =\left\{b(x) \in M \mid A_{2} \subseteq A+p(x)\right\}, \\
M(3) & =M \backslash M(2)=\left\{b(x) \in M \mid \exists g+G \mathbf{1} \in A_{2}, G(x) \neq 0\right\} \\
& =\left\{b(x) \in M \mid A_{2} \ddagger A+p(x)\right\} .
\end{aligned}
$$

Let $T \subset X$ be the set $T=\{x \in X \mid b(x) \in M(2)\}$. Set

$$
V=\left\{t \in \beta X \backslash X \mid \forall g+G \mathbf{1} \in A_{2}, G^{\beta}(t)=0\right\} .
$$


Thus $A_{2}$ is determined exactly as all those functions $g+G \mathbf{1}$ for which $G^{\beta}$ vanishes on $T \cup V \subset \beta X$ (i.e. $G$ vanishes on $T \subset X$, and $G^{\beta}$ on $\left.V \subset \beta X \backslash X\right)$, but where $g+G 1$ is arbitrary otherwise. The sets $V$ and $T$ are closed. Then

$$
M_{2}=\left\{b(t)+\left(p(t) \cap A_{2}\right) \mid t \in X \cup(\beta X \backslash V)\right\} .
$$

(Only those points of $\beta X$ are used in $M_{2}$ at which some function of $A_{2}$ does not vanish.) The maps $i_{1}, i_{2}$ are

$$
\begin{array}{lll}
i_{1}: M \rightarrow M_{2}, & i_{1}(b(x))=b(x)+p(x) \cap A_{2} & \text { for } x \in X ; \\
i_{2}: M_{2} \rightarrow M_{3} \cong \beta X, & i_{2}\left[b(t)+\left(p(t) \cap A_{2}\right)\right]=b(t)+p(t) & \text { for } t \in \beta X .
\end{array}
$$

In our previous notation for $m \in M$, we get

$$
\begin{array}{cc}
i_{1}[M(2)]=\{b(x) \mid x \in T\}, & \frac{A_{2}}{i_{1}(m)} \cong \mathrm{LCH} ; \\
i_{1}[M(3)]=\left\{b(x)+\left(p(x) \cap A_{2}\right) \mid x \in X \backslash T\right\}, \quad \frac{A_{2}}{i_{1}(m)} \cong C \times \mathrm{LCH} ; \\
m_{2} \in M_{2} \mid i_{1}(M)=\left\{A+\left(p(t) \cap A_{2}\right) \mid t \in \beta X \backslash X, \exists g+G \mathbf{G} \in A_{2} \text { with } G^{\beta}(t) \neq 0\right\}, \\
\frac{A_{2}}{m_{2}} \cong C ; \\
m_{3} \in M_{3} \mid i_{2} i_{1}(M)=\{A+p(t) \mid t \in \beta X \backslash X\}, \quad \frac{R+A}{m_{3}} \cong C .
\end{array}
$$

Since $X \cong i_{2} i_{1}(M) \subset M_{3}=\beta X$, it follows that $i_{1}(M) \subset M_{2}$ and $i_{2} i_{1}(M) \subset M_{3}$ are dense.

\section{REFERENCES}

1. R. C. Busby, Double centralizers and extensions of $C^{*}$-algebras, Trans. Amer. Math. Soc. 132 (1968), 79-99.

2. - Some remarks on the structure spaces and extensions of $C^{*}$-algebras, J. Functional Analysis 1 (1967), 370-377.

3. J. Dauns and K. H. Hofmann, The representation of rings by sections, Mem. Amer. Math. Soc. No. 83, 1968, pp. 1-180.

4. - Spectral theory of algebras and adjunction of identity, Math. Ann. 179 (1969), 175-202.

5. J. Dixmier, Ideal center of a $C^{*}$-algebra, Duke Math. J. 35 (1968), 375-382.

6. M. C. Flanders, Ideal $C^{*}$-algebras, Ph.D. Thesis, Tulane University, New Orleans, La., 1968.

7. B. E. Johnson, An introduction to the theory of double centralizers, Proc. London Math. Soc. 14 (1964), 299-320.

8. B. Mitchell, Theory of categories, Academic Press, New York, 1965.

9. H. H. Schaefer, Topological vector spaces, Macmillan, New York, 1966.

TULANE UNIVERSITY,

New Orleans, Louisiana 